\title{
1 Neural mechanisms driving hunger-induced changes in sensory perception and behavior in
}

2 Caenorhabditis elegans

4 Hiu E. Lau ${ }^{1,2}$, Zachary T. Cecere ${ }^{2,3,4}$, Zheng Liu ${ }^{2}$, Claire J. Yang ${ }^{2}$, Tatyana O. Sharpee ${ }^{3,4}$, and 5 Sreekanth H. Chalasani ${ }^{1,2,3}$

${ }^{1}$ Division of Biological Sciences, University of California, San Diego, La Jolla, CA 92093, 8 USA.

$9{ }^{2}$ Molecular Neurobiology Laboratory, The Salk Institute for Biological Studies, La Jolla, CA 1092037, USA.

$11{ }^{3}$ Neurosciences Graduate Program, University of California, San Diego, La Jolla, CA 92093, 12 USA.

$13{ }^{4}$ Computational Neurobiology Laboratory, The Salk Institute for Biological Studies, La Jolla, 14 CA, 92037, USA.

15 Author for correspondence: schalasani@ salk.edu

\section{Summary}

18 While much is known about how external cues affect neural circuits, less is known about how 19 internal states modify their function. We acutely food-deprived C. elegans and analyzed its

20 responses in integrating attractant and repellent signals. We show that food deprivation leads to a 21 reversible decline in repellent sensitivity; with no effect on appetitive behavior allowing animals 22 to engage in higher risk behavior. Multiple tissues including the intestine and body wall muscles 23 use a conserved transcription factor, MondoA, to detect the lack of food and release AEX-5 24 convertase processed peptides from dense core vesicles. Subsequently, ASI chemosensory 25 neurons use the DAF-2 insulin receptor and non-canonical signaling to integrate the tissue26 released peptide signals modifying their stimulus-evoked adaptation rate. We suggest that 27 altering ASI neuronal dynamics affects its function and modifies behavior. Together, these 28 studies show how internal state signals modify sensory perception and risk assessment to 29 generate flexible behaviors.

\section{Introduction}


Animals have evolved intricate mechanisms to detect relevant environmental cues as well as integrate information about various internal states including hunger and sleep (Sternson et al., 34 2013; Taghert and Nitabach, 2012). While progress has been made in decoding the neural 35 circuits processing environmental changes, less is known about the machinery that integrates 36 information about internal states. Of particular importance is nutritional status, which has a 37 profound effect on animal survival and elicits dramatic changes in food-seeking behaviors 38 (Atasoy et al., 2012). Moreover, multiple species have been shown to alter their behavior during periods of starvation (Gillette et al., 2000; Inagaki et al., 2014; Kawai et al., 2000; Sengupta, 2013). This is thought to be achieved by constant crosstalk between the central nervous system and the various peripheral organs (Dietrich and Horvath, 2013).

In mammals, signals from peripheral tissues (hormones and nutrients), most notably the gut-released polypeptide ghrelin, trigger neuronal changes and affect behaviors (Kojima et al., 1999; Tschop et al., 2000). Ghrelin acts on hypothalamic neurons that express agouti-related protein (AGRP) and neuropeptide Y (NPY) (Andrews et al., 2008; Cowley et al., 2003), as well as other brain regions to increase food intake (Carlini et al., 2004; Malik et al., 2008). In contrast, leptin and insulin peptides released from adipose tissue and the pancreas respectively, act to suppress feeding behavior (Air et al., 2002). Apart from feeding behaviors, these peptide signals also influence neural circuits regulating anxiety (Dietrich et al., 2015), indicating that this signaling has a broader role in modulating brain function. Moreover, malfunction of peripheral organ-nervous system communication axis results in a number of metabolic disorders, including

53 diabetes and obesity (Dietrich and Horvath, 2012; Muller et al., 2015). Despite the importance of 54 this physiological process, the precise neuronal machinery integrating metabolic signals and 55 modifying behaviors remains unknown.

The nematode Caenorhabditis elegans with just 302 neurons (White et al., 1986), 20 cells 58 in its intestine (McGhee, 2007) and 95 body wall muscle cells (Moerman and Williams, 2006) 59 provides a unique opportunity for a high-resolution analysis of how the nervous system 60 integrates internal signals. Previous studies have shown that C. elegans, similar to mammals, 61 exhibits a number of behavioral, physiological and metabolic changes in response to altered 62 nutritional status. When worms are removed from food, they exhibit a $50 \%$ reduction in their 
63 feeding rate (Avery, 1993). When returned to food, starved animals temporarily feed faster than 64 well-fed animals (Avery and Horvitz, 1990), suggesting that feeding is not a simple reflex to 65 food stimulus but can be modified by experience. Moreover, upon food deprivation, C. elegans 66 hermaphrodites will retain eggs (Trent et al., 1983), are unlikely to mate with males (Lipton et 67 al., 2004) and initiate altered foraging behaviors (Gray et al., 2005; Hills et al., 2004; Sawin et 68 al., 2000). Moreover, many molecules that signal hunger are conserved between C. elegans and 69 vertebrates. For example, neuropeptide Y (NPY) signaling influences feeding behaviors in 70 nematodes and mammals (de Bono and Bargmann, 1998; Mercer et al., 2011; Nassel and 71 Wegener, 2011). Similar effects are also seen with insulin and dopamine signaling, which seem 72 to act via modifying chemosensory activity and behavior in nematodes (Chalasani et al., 2010; 73 Ezcurra et al., 2011) and on mammalian hypothalamic and mid-brain circuits respectively (Air et 74 al., 2002; Berthoud, 2011; Figlewicz and Sipols, 2010) to modify feeding behavior.

Here we use $C$. elegans to dissect the machinery integrating internal food signals and modifying behaviors. We combined acute food deprivation with a behavioral assay that quantifies the animal's ability to integrate both toxic and food-related signals, mimicking a simplified real world scenario. In this sensory integration assay, animals cross a toxic copper

80 barrier (repellent) and chemotax towards a point source of a volatile odor, diacetyl (attractant)

81 (Ishihara et al., 2002). We show that food-deprived animals have reduced sensitivity to the

82 repellent and cross the copper barrier more readily than well-fed animals. Moreover, we find that 83 multiple internal tissues sense the lack of food, release peptides and use non-canonical insulin 84 signaling to alter the adaptation rate of chemosensory neurons. This altered neuronal state allows 85 animals to reduce their avoidance to repellents and undertake a higher risk strategy in their 86 search for food.

\section{Results}

89 Acute food deprivation specifically alters repellent-driven behaviors

90 Animals simultaneously integrate both attractant and repellent signals from their environment to 91 generate appropriate behavioral readouts. To mimic these interactions, animals are exposed to a 92 copper repellent barrier and a gradient of a volatile attractant, diacetyl (Ishihara et al., 2002). The 93 proportion of animals that cross the copper barrier are counted and expressed as an integration 
94 index (Figure 1A). We analyzed the behavior of well-fed wild-type animals and found that few

95 cross the copper barrier and locomote towards the spot of the diacetyl (white bar, Figure 1B,

96 Table S1 and Movie S1). In contrast, when animals are food-deprived for 3 hours, we observe an

$97 \sim 100 \%$ increase in the number of animals crossing the copper barrier (dark bar, Figure 1B, Table

98 S1 and Movie S2). Moreover, we found that food-deprived animals cross the repellent barrier 99 throughout the entire 45-minute assay suggesting a broad change in behavior (Figure S1A).

100 Food-deprived animals also do not alter locomotory speed indicating that their general

101 movement is not modified (Figure S1B). Also, this effect is not specific to diacetyl, as food-

102 deprived animals also cross the copper barrier more when paired with other volatile attractants,

103 isoamyl alcohol or benzaldehyde (Figure S1C). The proportion of animals crossing the copper

104 barrier in both well-fed and food-deprived states is a function of both attractant and repellent 105 concentrations (Figures S1D-E and Table S1). Together, our results show that food-deprived 106 animals cross the repellent barrier more readily than well-fed animals.

Next, we tested whether food deprivation affects the sensitivity to repellents or attractants independently. We analyzed the behavior of well-fed and food-deprived animals on assay plates

110 with either copper or odor gradients alone. We found that food-deprived animals crossed the 111 copper barrier more readily than well-fed animals, suggesting that their responsiveness to copper 112 is reduced (Figure 1C, Figure S1F and Table S1). In contrast, food-deprived animals did not alter 113 their attraction to diacetyl or other volatile attractants (Figure 1C and Figure S1G). Given the 114 small number of animals that cross the copper barrier alone (Figure 1B, a high \% increase from 115 well-fed value results from low integration index in the well-fed condition, Table S1), we 116 continued to pair copper with the diacetyl attractant for further analysis. We found that food117 deprived animals have reduced sensitivity to copper, intermediate concentrations of fructose and 118 salt, but not quinine, 2-nonanone and other concentrations of fructose and salt (Figure S1H). We 119 suggest that copper and these intermediate concentrations of salt and fructose are environmental 120 cues that $C$. elegans might have evolved to detect and reducing sensitivity to these cues enables 121 the animal to use a higher risk food search strategy.

122

\section{Food deprivation reversibly modifies animal behavior}


124 We probed the time course of the food-deprivation effect on animal behavior. We found that

125 animals need to be food deprived for at least 2 hours before they showed a reduction in their

126 copper sensitivity with a maximum effect at 3 hours (Figure 1D). We also found that animals did

127 not deplete their fat stores during the 3-hour food deprivation (Figure S2A-D), suggesting that

128 this behavior change is likely to be independent of fat metabolism. Next, we asked whether the

129 food-deprivation effect was reversible. We food-deprived animals for 3 hours and then returned

130 them to food for different durations and analyzed animal behavior after the food experience. We

131 found that food-deprived animals that had been returned to food for 5 hours reverted their copper

132 avoidance behavior to well-fed behavior (Figure 1E). These results indicate that food deprivation

133 reversibly reduces copper avoidance.

136 changes in food distribution, oxygen and carbon dioxide concentrations, small molecule

137 metabolites and others (Calhoun et al., 2015; Carrillo and Hallem, 2015; Ludewig and 138 Schroeder, 2013). To uncouple the tactile and chemosensory input of the bacteria [C. elegans 139 consume E. coli (Brenner, 1974)] from the nutritional value of ingesting bacteria, we analyzed 140 the effect of modified bacteria on animal behavior. Aztreonam is a drug that inhibits bacterial 141 cell wall synthesis and results in long filaments of bacteria that do not divide and cannot pass 142 through the grinder into the $C$. elegans intestine (Gruninger et al., 2008). Animals exposed to 143 aztreonam-treated bacteria experience the tactile and chemosensory input, but are unlikely to 144 derive nutritional value from the food. We found that exposing animals to drug-treated bacteria 145 for 3 hours was sufficient to reduce their sensitivity to copper, suggesting that the lack of food in 146 the animal alters copper sensitivity (Figure 1F). Together, these results show that the lack of food

147 in the $C$. elegans intestine, but not the absence of sensory cues, reduces the animal's sensitivity 148 to copper.

150 Food deprivation reduces reorientations enabling more animals to cross the repellent 151 barrier

152 To analyze how food deprivation modifies animal behavior, we developed a new multi-worm 153 tracker. This tracker enables us to identify individual animals and their trajectories over many 154 minutes as they integrate repellent and attractant signals in the sensory integration assay (see 
155 Extended Experimental Procedures for details of the tracker). We tracked animals in both well-

156 fed and food-deprived conditions and filtered the tracking data to consider a time window

157 between 3 and 15 minutes in the assay. Additionally, we included events executed by animals

158 moving towards the copper barrier. We found that food-deprived animals are more likely to cross

159 the repellent barrier when compared to well-fed animals as shown by the increased density of

160 well-fed animal tracks before the copper barrier (Figure 2A, 2B). We performed a similar

161 analysis on animals moving away from the copper barrier and found no significant differences

162 between well-fed and food-deprived conditions (data not shown). We then fit a Laplace mixture

163 model for the distribution of turn events observed at different distances from the copper barrier

164 (Figure S3A-D). This allows us to precisely quantify the reorientations and small turns as the

165 animals approached the copper barrier and after crossing the barrier (see Extended Experimental

166 Procedures for quantification details). While a large reorientation allows the animal to reverse its

167 direction of movement, small turns generate smaller changes in the direction (Figure S3A-D).

168 These large reorientations and small turns are similar to the previously described "pirouettes" 169 and "small turns" respectively (Iino and Yoshida, 2009; Pierce-Shimomura et al., 1999).

170 Compared to food-deprived animals, we found that well-fed animals make significantly more

171 reorientations as they approach the copper barrier (Figure 2C). In contrast, there is no difference

172 between well-fed and food-deprived animals in their relative small turn probabilities (Figure

173 2D). Since our assays included a paralytic, we were unable to obtain movement data from

174 animals close to diacetyl. Together, these results suggest that well-fed animals reorient more,

175 thus avoiding the copper barrier, while food-deprived animals reorient less and cross the copper

176 barrier more frequently. Moreover, these data also suggest that food-deprived animals are

177 unlikely to avoid toxic copper compounds in their environment allowing them to execute food

178 search strategies with higher risk.

180 Intestine and body wall muscles use MML-1, but not MXL-2 to sense the lack of food and 181 releases peptides to signal to the neurons

182 Our studies show that the lack of food inside the animal is responsible for the transient reduction

183 in copper sensitivity. We hypothesized that internal tissues sense this lack of food and signal to

184 the nervous system to modify neuronal function and behavior. To gain insights into how these

185 tissues sense the absence of food, we used a candidate gene approach. In mammalian cells, 
186 glucose is rapidly converted to glucose-6-phostphate, whose levels are sensed by a two basic187 helix-loop-helix-leucine zipper transcription factors, MondoA and ChREBP (Carbohydrate 188 Response Element Binding Protein). In well-fed conditions, MondoA binds the excess glucose189 6-phosphate and Mlx (Max-like protein X) and translocates to the nucleus where it activates 190 transcription of glucose-responsive genes. In the absence of glucose, MondoA remains in the 191 cytoplasm (Havula and Hietakangas, 2012; Stoltzman et al., 2008). C. elegans homologs for 192 MondoA and Mlx have been identified as MML-1 and MXL-2 respectively (Grove et al., 2009).

193 Furthermore, MML-1 has also been shown to translocate into the intestinal nuclei under well-fed 194 conditions (Johnson et al., 2014). We predicted that $m m l-1$ mutants would be unable to sense the 195 lack of food and thereby unable to reduce copper sensitivity after food deprivation. Consistently, 196 we found that $m m l-1$, but not $m x l-2$ mutants are defective in their integration responses after food 197 deprivation (Figure 3A). To localize the MML-1 function to a specific tissue, we expressed the 198 full-length coding sequence under specific promoter elements and analyzed its effect on 199 behavior. We used promoter elements that drive expression in all neurons (H2O), intestine ( $g l y$ 200 19) or body wall muscles (myo-3) (Figure 3B) (Okkema et al., 1993; Shioi et al., 2001; Warren et 201 al., 2001). We find that expressing the full-length cDNA encoding MML-1 specifically in the 202 intestine and body wall muscles, but not neurons is sufficient to restore normal behavior to $\mathrm{mml}$ 2031 mutants (Figure 3A). We suggest that in the absence of food, MML-1 remains in the cytoplasm 204 of intestinal and body wall muscle cells and speculate that the cytosolic MML-1 reduces copper 205 sensitivity by modifying signaling between tissues.

Next, we hypothesized that the intestine and body wall muscles release peptide signals to 208 relay the lack of food signal to the nervous system. To identify the relevant class of peptides, we 209 analyzed gene mutants in peptide processing. The C. elegans genome encodes four known pro210 protein covertases (AEX-5, EGL-3, BLI-4, and KPC-1) that cleave an overlapping subset of pro211 peptides to generate mature peptides, which are further modified and packed into dense core 212 vesicles ( $\mathrm{Li}$ and $\mathrm{Kim}, 2008$ ). Upon activation, dense core vesicles are released using the CAPS 213 protein (calcium activated protein for secretion, unc-31) (Figure 3C) (Speese et al., 2007). We 214 found that aex-5 mutants were specifically defective in their sensory integration response under 215 food-deprived, but not well-fed condition (Figure 3D, Table S1). In contrast, egl-3 mutants were 216 defective in their sensory integration behavior in both well-fed and food-deprived conditions, 
217 while bli-4 and $k p c-1$ were defective only in the well-fed state (Figure 3D and Table S1).

218 Previously, neuropeptide signaling has been shown to play a role in modifying sensory

219 integration behavior (Ishihara et al., 2002) and we suggest that peptides processed by EGL-3,

220 BLI-4 and KPC-1 might be involved. Together these results show that AEX-5-processed

221 peptides are specifically required for animals to reduce their copper sensitivity after food

222 deprivation.

223

To localize AEX-5 function to a specific tissue, we expressed the full-length coding sequence under specific promoter elements that drive expression in all neurons (unc-119), 226 intestine (gly-19), body wall muscles (myo-3), pharynx (myo-2) and tail (lin-44) in the null 227 mutant background and analyzed its effects on behavior (Figure 3B) (Hilliard and Bargmann, 228 2006; Maduro and Pilgrim, 1995; Okkema et al., 1993; Warren et al., 2001). We found that 229 restoring AEX-5 to either the intestine, body wall muscles or neurons but not the pharynx or tail 230 was sufficient to revert aex-5 null mutants to normal modulation after food deprivation (Figure 231 3E). To confirm AEX-5 function in intestine, body wall muscles and neurons, we knocked down 232 this gene specifically in those tissues and analyzed the effects on integration behavior. 233 Expressing sense and anti-sense transcripts under cell-selective promoters has been shown to 234 knock down the gene of interest in the target cells (Esposito et al., 2007; Leinwand and 235 Chalasani, 2013). Using this approach, we found that knocking down aex-5 in the intestine, body 236 wall muscles or neurons generated animals that were defective in altering behavior after food237 deprivation (Figure 3F). Previously, AEX-5 was shown to function in the intestine to modulate 238 defecation behavior and body wall muscles to affect neuromuscular junction function (Mahoney 239 et al., 2008; Sheng et al., 2015). However, our results showing AEX-5 activity in neurons are 240 novel. Also, we found that knocking down UNC-31 [CAPS protein required for peptide release 241 (Speese et al., 2007)] in the intestine, body wall muscles or neurons also rendered animals unable 242 to modify their response in the integration assay after food deprivation (Figure 3G). Taken

243 together, these results suggest that while AEX-5 processing is required in the intestine, body wall 244 muscles and neurons, release of AEX-5 processed peptides from any of those tissues is sufficient 245 to reduce copper sensitivity after food deprivation. Moreover, our results also show that the 246 intestine and body wall muscles also use dense core vesicles to release peptides, a novel 247 mechanism. 
ASI chemosensory neurons use DAF-2 receptors to integrate intestine-released AEX-5 processed peptide(s)

251 To gain insights into the nature of the intestine-released peptide signal, we analyzed mutants in 252 downstream receptors. The $C$. elegans genome encodes at least 122 neuropeptide genes 253 including 42 neuropeptide like proteins (NLPs), FMRFamine-related peptides (FLPs) and 40 254 insulin-like peptides (ILPs) (Hobert, 2013). Many of the NLPs and FLPs are thought to act on G255 protein coupled receptors (GPCRs), while ILPs bind the receptor tyrosine kinase, DAF-2 to 256 influence cellular functions (Chen et al., 2013b; Hobert, 2013; Leinwand and Chalasani, 2013;

257 Pierce et al., 2001). Upon binding cognate ligand(s), GPCRs use heterotrimeric G $\alpha \beta \gamma$ proteins to 258 activate signal transduction (Hamm, 1998). There are two $\mathrm{G} \gamma$ subunits, $g p c-1$ and $g p c-2$ in the $C$. 259 elegans genome, both with viable null mutants (Jansen et al., 1999). We found that mutants in 260 the insulin receptor, DAF-2, were defective in their response to food deprivation, suggesting that 261 the intestine and other tissues might release insulin-like peptide(s) to modify integration response 262 after food deprivation (Figure 4A). In contrast, mutants in the two $G \gamma$ subunits or the 263 carboxypeptidase, EGL-21, which is required to generate mature NLPs and FLPs (Husson et al., 264 2007) are not defective in their responsivity to copper after food deprivation (Figure S4A). 265 Together, these data suggest that insulin signaling might be involved in reducing copper 266 sensitivity after food deprivation.

To localize the site of DAF-2 action, we analyzed the effect of rescuing this receptor in different tissues. We found that expressing daf-2 under the neuronal, but not intestine or body wall muscle promoters (Hung et al., 2014) restored normal behavior to the daf-2 mutants (Figure 4A). These results implied that AEX-5 convertase might process an insulin-like peptide(s) in the

272 various internal tissues. To identify the cognate ligand(s), we analyzed null mutants or RNA 273 interference knockdowns against each of the 40 insulin-like peptides (Hobert, 2013). However, 274 we found that none of the gene mutants or knockdowns in these peptides affected the altered 275 integration response upon food deprivation (Table S2). We speculate that a combination of 276 insulin-like or other peptide(s) might relay the lack of food signal from the intestine and other 277 tissues. Taken together, these results suggest that neuronally expressed DAF-2 receptors might 278 detect AEX-5 processed peptides that are released from multiple internal tissues. 
To localize DAF-2 function to individual neurons, we used cell-selective promoters and generated transgenic animals. Previous studies have shown that three pairs of chemosensory neurons ASI, ASH and ADL detect copper ions and generate avoidance response (Hilliard et al., 2002). Given that food deprivation alters the animal's response to copper ions specifically, we tested whether DAF-2 was required in any of these sensory neurons. We found that restoring DAF-2 to ASI, but not ASH or ADL was sufficient to restore normal behavior to daf-2 mutants (Figure 4B, Table S1). ASI-specific expression does not completely rescue the food-deprivation driven daf-2 mutant behavioral phenotype suggesting that DAF-2 might also be required in additional neurons. Together, these results suggest that ASI neurons use DAF-2 receptors to detect internal tissue-released peptide(s) to modify integration behavior.

ASI neurons use non-canonical insulin signaling to integrate AEX-5 processed peptide 292 signals

293 To identify the components of the DAF-2 signaling in ASI neurons that integrate food status 294 signals, we analyzed gene mutants in candidate pathway components (Figure 4C). We observed 295 that mutants in the canonical insulin-signaling pathway components phosphoinositide 3-kinase 296 (PI3K, age-1), serine/threonine kinases AKT-1, AKT-2 (akt-1, akt-2), 3-phosphoinositide297 dependent kinase $1(p d k-1)$ and lipid phosphatase (daf-18, PTEN suppressor) performed 298 normally in the sensory integration assay after food deprivation (Figure S4B) (Lapierre and 299 Hansen, 2012). In contrast, mutants in serum and glucocorticoid inducible kinase-1 (sgk-1) are 300 defective in their copper sensitivity after food deprivation (Figure 4D). We found that restoring 301 SGK-1 function to ASI neurons specifically was sufficient for normal integration response in 302 food-deprived $s g k-1$ mutants (Figure 4D). SGK-1 has been previously shown to interact with the 303 mTORC2 complex including Rictor (Jones et al., 2009; Mizunuma et al., 2014). We also tested 304 mutants in rict-1 (C. elegans Rictor) in our sensory integration assay and found that these 305 mutants are also unable to alter their behavior after food deprivation (Figure 4D). Similar to 306 SGK-1, we found that RICT-1 was also required in ASI neurons to restore normal food307 deprivation behavior to rict-1 mutants (Figure 4D). These results suggest that SGK-1 and RICT3081 might function in the same pathway downstream of DAF-2 receptors in ASI neurons. These 309 results are consistent with previous studies showing that SGK-1 and mTORC2 act parallel to the 
310 canonical insulin-signaling pathway to regulate stress responses and animal lifespan (Hertweck

311 et al., 2004). Collectively, these results show that both SGK-1 and RICT-1 function in ASI

312 neurons downstream of DAF-2 receptors to reduce copper sensitivity after food deprivation.

To test whether AEX-5 and DAF-2 function in the same pathway, we performed genetic epistasis experiments. We generated an aex-5;daf-2 double mutant, which did not show any 316 additional defects when compared to either aex-5 or daf-2 single mutant (Figure 4E). We also

317 found that expressing AEX-5 in the intestine and DAF-2 in ASI sensory neurons restored normal 318 integration response after food deprivation (Figure 4E). Together, these data show that the 319 intestine released AEX-5 processed peptides are detected by ASI neurons using the DAF-2 320 receptors. These results suggest the following order for these signaling events: food deprivation 321 leads to lack of food within the animal, which is detected by the intestine and body wall muscles 322 using cytosolic MML-1 leading to the release of AEX-5 processed peptides that bind DAF-2 323 receptors and are processed by downstream SGK-1 and RICT-1 in ASI and other neurons to 324 reduce copper sensitivity and alter behavior.

\section{Food-deprivation alters ASI neuronal adaptation rate}

327 To test how food deprivation modifies neural function, we probed the activity of copper-sensitive 328 neurons using calcium imaging. We localized a genetically encoded calcium indicator 329 (Akerboom et al., 2012) to the nuclei of ASI and ASH neurons, allowing us to measure neural 330 activity in both neurons simultaneously. We also expressed the fluorescent mCherry protein 331 under an ASI-selective promoter in the same transgenic animal enabling us to specifically 332 identify ASI neuronal nuclei (Figure S5A-C). Recently, nuclear calcium dynamics have been 333 shown to be similar to those measured from the cytoplasm (Schrodel et al., 2013), validating our 334 approach. We presented the repellent copper solution to the nose of an animal constrained in a 335 microfluidic device and recorded neural activity as previously described (Chalasani et al., 2007) 336 in both well-fed and food-deprived conditions. We found that both well-fed and food-deprived 337 animals responded similarly to long pulses (30 seconds) of repellent stimuli (data not shown).

338 We then presented the repellent stimulus as 1-second pulses (1 second on, 1 second off) for 30 339 seconds. This protocol allows us to probe the activity of ASI and ASH neurons to repeated 340 pulses of copper solution, a regimen that animals might experience as they encounter the copper 
341 barrier (see Experimental Procedures). We found that well-fed ASI neurons responded to the

342 removal of copper stimuli with increasing fluorescence changes until a maximal fluorescence

343 change (peak). Subsequently, the ASI calcium signals dropped suggesting that this neuron had

344 adapted to the copper stimuli (black line indicates average, Figure 5A). This result is consistent

345 with previous studies showing that adapted chemosensory neurons do not respond to stimuli

346 (Chalasani et al., 2010). In contrast, under food-deprived conditions, ASI neurons had a higher

347 threshold requiring additional stimulus pulses to observe a calcium change. Moreover, we did

348 not observe a distinct maximal fluorescence or a decline in the calcium signal, suggesting that

349 ASI neurons did not adapt (green line indicates average, Figure 5A). Moreover, in both well-fed

350 and food-deprived conditions, we observe an ASI calcium response to the removal of last copper

351 stimulus suggesting that there are additional calcium dynamics. Given that we observe food-

352 deprivation modifies animal behavior as the animal experiences the copper barrier, we focused

353 our analysis on the calcium dynamics obtained in response to the repeated stimuli. We quantified

354 the average change in fluorescence in an 8-second window both before and after the peak in

355 well-fed conditions and compared the data to a similar time window in the food-deprived

356 conditions (Figure 5B). We also tested ASI responses to additional copper stimulus

357 concentrations and observed similar dynamics at $25 \mathrm{mM}$ and $100 \mathrm{mM}$, but not at $10 \mathrm{mM}$ (Figure

358 S6A, S6C and S6E). These data suggest that ASI neurons show a dose-dependent response to the

359 removal of copper stimuli and can adapt to repeat stimulus pulses, which is altered by food

360 deprivation. Together, these data show that food deprivation increases the stimulus threshold and

361 transforms ASI from an adapting to a non-adapting state.

362

Next, we analyzed ASH activity data in both well-fed and food-deprived conditions. We

364 found that ASH neurons responded to both the addition and removal of copper stimuli. Under

365 well-fed conditions, we observed complex dynamics including an increase in calcium signal to

366 the initial stimulus pulses followed by responses without change in baseline through the middle

367 stimulus pulses and finally, a smaller decline in the baseline response to the last few stimulus

368 pulses (black line indicates average, Figure 5C). We also find that food deprivation lowers the

369 ASH threshold (higher response to the initial stimulus pulses), which is followed by responses

370 without change in baseline through the middle stimulus pulses and no decline in the baseline

371 response to the last few stimulus pulses (green line indicates average, Figure 5C). Similar to ASI 
372 dynamics, we also observe ASH responses to removal of the last copper stimulus pulses 373 suggesting additional dynamics. We performed a similar analysis on the ASH calcium data and 374 found that food deprivation reduces the stimulus threshold and had a smaller effect on the ASH 375 adaptation rate (Figure 5D). We also tested additional copper stimulus concentrations and found 376 that ASH neurons also show dose-dependent responses to both addition and removal, but are not 377 significantly affected by food deprivation. Together, these data show that food deprivation 378 reduces the stimulus threshold of ASH neurons and likely affects additional stimulus dynamics.

Our genetic experiments showed that food-deprivation effects are lost in daf-2 mutants.

381 To test whether the changes in ASI neural activity also use the same genetic pathway, we

382 performed a similar analysis of their copper-evoked responses in daf-2 mutants. Consistent with 383 our genetic analysis, we found that the ASI-specific neural activity changes after food 384 deprivation are lost in the $d a f-2$ mutants (Figure 6A-6D). We also compared the ratio of change 385 in food-deprived to well-fed conditions in both wild-type and daf-2 mutants and found that the 386 insulin-receptor mutant had a significant effect on ASI, but not ASH activity (Figure 6E). 387 Collectively, these results show that food deprivation affects ASI threshold and adaptation 388 kinetics in a DAF-2 dependent manner.

\section{Discussion}

391 We used food deprivation in C. elegans as a model to understand how changes in internal states

392 modify behavior. We show that food-deprived animals reversibly alter their behavior by 393 reducing their repellent responsiveness, allowing them to traverse potentially toxic environments 394 in their search for food. Multiple tissues including the intestine, body wall muscles and neurons 395 independently sense the lack of food and release peptide signals that are integrated by DAF-2 396 receptors on ASI neurons (Figure 6F). This novel, non-neuronal, dense core vesicle release 397 dependent peptide signaling transforms ASI neurons from a rapidly adapting to a non-adapting 398 neuron modifying animal behavior. We suggest that altering the state of sensory neurons affords 399 greater dynamic range of control over behavioral decisions. More generally, we demonstrate how 400 neuronal activity is regulated by internal state signals to modify behaviors lasting many minutes.

\section{Multiple tissues release peptide(s) to signal "lack of food"}


403 Multicellular animals sense and regulate glucose homeostasis at several levels. While insulin and

404 glucagon maintain constant levels of circulating glucose, the Myc-family transcription factors are 405 used within cells. Glucose uses cell-membrane localized transporters to enter cells, where it is 406 rapidly converted into glucose-6-phosphate (Jordan et al., 2010). This intermediate metabolite is 407 sensed by the Myc-Max complex, which binds glucose-6-phosphate and translocates to the 408 nucleus where it regulates the transcription of glucose-responsive genes (Havula and 409 Hietakangas, 2012). While the role of ChREBP/MondoA-Mlx-glucose-6-phosphate complex in 410 regulating the gene transcription is well studied (Li et al., 2010; Stoeckman et al., 2004; 411 Stoltzman et al., 2008), the role of these proteins in the cytoplasm remains poorly understood. 412 We show a specific role for MML-1 (MondoA homolog), but not MXL-2 (Mlx homolog) in the 413 intestine and body wall muscles in reducing copper sensitivity after food deprivation. We suggest 414 that in the absence of MML-1, these two tissues are unable to detect the lack of glucose and do 415 not relay signals to modify the downstream neuronal circuits. Moreover, we also speculate that 416 MML-1 (MondoA) accumulation in the cytoplasm (in the absence of glucose) enables the 417 intestine and body wall muscle cells to release peptide(s) relaying a "lack of glucose" signal to 418 other tissues.

Our analysis describes a role for the pro-protein convertases in modifying behavior upon 421 food deprivation. We show that while egl-3, bli-4 and $k p c-1$ mutants are defective in their 422 sensory integration response, aex-5 mutants are specifically affected in their response to food 423 deprivation. Moreover, we show that AEX-5 functions in the intestine, body wall muscles and 424 neurons to process relevant peptide(s). Previously, aex-5 has been shown to function in both the 425 body wall muscle cells and the intestine to regulate exocytosis at the neuromuscular junction and 426 the defecation motor program respectively (Doi and Iwasaki, 2002; Sheng et al., 2015). 427 However, our studies confirming a role for AEX-5 in neurons are novel. We suggest that $C$. 428 elegans neurons use a MML-1 independent mechanism to detect the lack of glucose and release 429 AEX-5 processed peptide(s). Surprisingly, we find that restoring AEX-5 function to the intestine, 430 the body wall muscles or neurons is sufficient to restore normal behavior to aex-5 mutants, while 431 knocking down this gene in any of these three tissues disrupts wild-type behavior. Together, 432 these results suggest that intestine, body wall muscle or neuronal release of AEX-5 processed 433 peptide(s) alone is sufficient to relay food status signals and this signaling is required in all three 
434 tissues in wild-type animals. Given that our transgenic rescue experiments involve expressing 435 more than one copy of the AEX-5 protease in the three tissues (see Extended Experimental 436 Procedures), we speculate that this "food status" signaling is regulated at the level of pro-peptide 437 cleavage such that excess mature peptide(s) from the intestine, body wall muscles or neurons can 438 compensate for the lack of signal from the other two tissues.

Moreover, we also identify a role for the dense core vesicle release machinery in nonneuronal tissues including the intestine and body wall muscles. Interestingly, in mammals a second CAPS protein (CAPS2) that is expressed in non-neuronal tissues has been identified

443 (Speidel et al., 2003). These results suggest that peptide release from non-neuronal tissues might

444 also involve dense core vesicles across multiple species from worms to mammals. Collectively, 445 we show that multiple tissues including the intestine, body wall muscles and neurons process 446 precursors using the AEX-5 protease and release mature peptide(s) using the CAPS protein 447 relaying "food status" signals to downstream neurons.

\section{ASI chemosensory neurons integrate "food status" signals}

450 Our studies show that while the intestine, body wall muscles and neurons release AEX-5 451 processed peptide(s), ASI chemosensory neurons use the tyrosine kinase insulin receptor (DAF-

452 2) to integrate these signals. Three lines of evidence suggest that the internal tissues are releasing 453 insulin-like peptide(s)- first, the food-deprivation effect does not require G-protein receptor 454 signaling, second, the insulin receptor (DAF-2) integrates these signals and third, DAF-2 and 455 AEX-5 function in the same pathway. Although our efforts to identify the cognate insulin-like 456 peptide (ILP) have been unsuccessful, we suggest that combination of ILPs might relay "food 457 status" signals from the intestine, body wall muscles and neurons to ASI sensory neurons. We 458 also find that non-canonical signaling pathway components are used downstream of DAF-2 to 459 integrate these peptide signals. Our results show that while PI-3Kinase, AKT kinase -1 and -2 , 460 PDK-1 and PTEN are not required, SGK-1 (serum and glucocorticoid inducible kinase) and its 461 binding partner Rictor (a component of the mTORC2 complex) are required to integrate AEX-5 462 processed peptide signals. Although SGK-1 has been shown to phosphorylate DAF-16 (FOXO) 463 in vitro (Hertweck et al., 2004), it might also indirectly regulate a subset of the DAF-16 target 464 genes (Chen et al., 2013a; Murphy and $\mathrm{Hu}, 2013$ ). We suggest that this SGK-1-Rictor 
465 (mTORC2) complex might integrate signals from multiple pathways including DAF-2 signaling 466 to regulate DAF-16-target genes (Mizunuma et al., 2014) and modify neuronal functions and 467 animal behavior.

Our genetic analysis shows that ASI chemosensory neurons integrate peptide signals and modify integration response after food deprivation. ASI neurons have been previously shown to

471 detect food signals to modify animal behavior (Calhoun et al., 2015; Gallagher et al., 2013).

472 However, our results showing that ASI integrates internal food status signals are novel.

474 Food-deprivation alters the ASI neural adaptation rate

475 Our imaging experiments show that ASH neurons respond to the copper pulses, before ASI 476 neurons, suggesting that ASH might be a low-threshold, while ASI is a high-threshold copper 477 detector. This is consistent with previous results showing that ASH is crucial for copper 478 avoidance (Hilliard et al., 2005; Hilliard et al., 2002) and with data showing that ASI is a high479 threshold detector for food (Calhoun et al., 2015). This dual coding strategy with high and low480 threshold neurons is commonly used across C. elegans and others species to efficiently encode 481 stimulus information (Calhoun et al., 2015; McGlone and Reilly, 2010). Moreover, our analysis 482 of ASH and ASI neural activity in daf-2 mutants reveals a surprising feature. We find that ASH 483 responses are greatly reduced in the daf-2 mutants, but spare some neural dynamics, particularly 484 in the early stimulus pulses. In contrast, the dynamics of the high-threshold ASI neurons are 485 significantly altered. We suggest that the food-deprivation signal has a stronger influence on the 486 high-threshold ASI, while sparing some of the signaling from the low threshold ASH neurons. 487 These results are also consistent with our genetic experiments showing that DAF-2 is specifically 488 required in ASI, but not ASH neurons to integrate internal food status signals.

We also show that food deprivation alters the adaptation kinetics in ASI sensory neurons and reduces its responsiveness to copper stimuli. Adaptation is a fundamental property of many 493 et al., 2013; Wark et al., 2009). Sensory neurons have been shown to adapt to a variety of 494 stimulus distributions and efficiently encode information (Wark et al., 2007). Indeed, individual 495 neurons in the rodent sensorimotor cortex and retinal ganglion cells and others have been shown 
496 to alter their input-output properties based on the size of the input stimulus and local statistical

497 context (Mease et al., 2013; Wark et al., 2009). We observe a similar change in the ASI neural 498 activity, where it adapts to repeated stimulus pulses allowing them to read local concentration 499 gradients more effectively. In contrast, under food-deprived conditions, the adaptation rate is 500 reduced making these neurons insensitive to changes in copper stimuli. Moreover, we also show 501 that non-canonical insulin signaling mediates some aspects of food deprivation by altering neural 502 adaptation rates leading to flexible behaviors. We suggest that similar peptide signaling 503 pathways might exist across different animal species allowing their nervous systems to integrate 504 internal information like food deprivation, stress and others.

We speculate that changes in ASI neuronal properties might underlie altered animal behavior. We observe that food-deprived animals have fewer reorientations as they approach the copper barrier allowing them cross the repellent and approach the attractant. In contrast, well-fed animals have higher number of reorientations allow them to avoid the copper barrier. We suggest

510 that transforming ASI from a rapidly adapting to a non-adapting neuron prevents this neuron

511 from detecting local changes in copper leading to fewer reorientations and allowing the food-

512 deprived animals to cross the copper barrier. These data are also consistent with previous studies

513 showing that ASI neurons suppress reorientations indicating that animals with high ASI activity

514 will re-orient less (Gray et al., 2005). These studies link metabolite sensing by internal tissues

515 with non-canonical insulin signaling that alters neuronal adaptation rate to modify chemosensory

516 behavior, a mechanism likely conserved across species.

\section{Experimental Procedures}

519 Standard culture, molecular biology and injection methods were used; details and strain 520 genotypes; tracker and calcium imaging data analysis are in Extended Experimental Procedures.

\section{Behavior Assays}

523 Control and food-deprived animals were grown to adulthood on regular nematode growth 524 medium (NGM) plates before they were washed and transferred to new food or food-free plates

525 respectively for the indicated duration. Sensory integration assay was performed on $2 \%$ agar 526 plates containing $5 \mathrm{mM}$ potassium phosphate $(\mathrm{pH} 6), 1 \mathrm{mM} \mathrm{CaCl} 2$ and $1 \mathrm{mM} \mathrm{MgSO}$. Repellent 
527 gradients (including $\mathrm{CuSO}_{4}$, glycerol, $\mathrm{NaCl}$, and quinine) were established by dripping $25 \mu \mathrm{l}$ of 528 solution across the midline of the plate (Ishihara et al., 2002). Copper gradients were visualized 529 using dyes (see Extended Experimental Procedures). Prior to the assay, the animals were washed 530 from the food or food-free plates before being transferred to the assay plates. After 45 minutes or

531 at indicated times, the integration index was computed as the number of worms in the odor half 532 of the plate minus the worms in origin half of the plate divided by the total number of worms that 533 moved beyond the origin. The percent increase of food-deprived animals from well-fed controls 534 was calculated by subtracting the averaged well-fed integration indices from each food-deprived 535 integration index, divided by the averaged well-fed integration index. Nine or more assays were 536 performed on at least three different days. Two-tailed unpaired $t$ tests (for Figure 1B only) and 537 one sample $t$ tests were used to obtain statistical information across food conditions. An ordinary 538 one-way ANOVA test was used to compare all conditions in Figures 1D -E. To compare across 539 strains for genetic rescue or knock down experiments, unpaired $t$ test with Welch's correction 540 was used.

541 Aztreonam-treated bacteria were prepared as previously described (Gruninger et al., 542 2008). E. coli was grown at $37^{\circ} \mathrm{C}$ overnight while shaking vigorously to an optical density (OD) 543 of less than 0.6 to avoid log phase. This culture was treated with aztreonam antibiotic (Sigma) at 544 a final concentration of $10 \mu \mathrm{g} / \mathrm{ml}$ and incubated at $37^{\circ} \mathrm{C}$ for 3 hours with minimal shaking at 70 545 RPM. Aztreonam-treated bacteria were immediately spread on plates also with $10 \mu \mathrm{g} / \mathrm{ml}$ 546 aztreonam, dried and used that day for behavior experiments.

\section{Calcium Imaging}

549 C. elegans expressing nuclear-localized GCaMP5K calcium indicators (Akerboom et al., 2012) 550 under an ASH and ASI selective promoter are trapped and imaged using a PDMS based 551 microfluidic device (Chalasani et al., 2007). Expressing mCherry protein in the cytoplasm under 552 an ASI-specific promoter identified ASI neurons. Both ASI and ASH neurons were imaged 553 simultaneously. M13 buffer (30mM Tris- $\mathrm{HCl} \mathrm{pH} 7.0,100 \mathrm{mM} \mathrm{NaCl}$ and $10 \mathrm{mM} \mathrm{KCl})$ were used 554 in all imaging experiments to prevent $\mathrm{CuSO}_{4}$ from precipitating. Prior to data collection, $\mathrm{ASH}$ 555 neurons were exposed to blue light for two minutes as previously published (Hilliard et al., 556 2005). Each animal was only imaged once using the 1-second on, 1-second off pulse protocol. 557 Images were captured using Metamorph software on an inverted microscope using a 
558 Photometrics EMCCD camera. Baseline F0 was measured as average fluorescence during a

559 three-second window (1s-4.4s). The ratio of change in fluorescence to the baseline $\mathrm{F}_{0}$ is plotted

560 using custom MATLAB scripts.

561

\section{$562 \quad$ Fat quantification}

563 Oil red O staining was conducted as previously described (Soukas et al., 2009). Briefly, 564 synchronized animals under well-fed or food-deprived animals were kept on ice for 10 minutes 565 to stop pharyngeal activity. After fixation, animals were taken through three freeze-thaw cycles 566 and exposed to a working solution of oil red $\mathrm{O}$ stain (40\% water: $60 \%$ oil red $\mathrm{O}$ in isopropanol). 567 Animals were imaged with a 20X objective on a Zeiss Axio Imager and an Orca Flash 4.0 568 camera. In all cases, staining in the intestine within 500 pixels from the bulb was quantified 569 using ImageJ (NIH). Percent of stained pixels was calculated using ImageJ, which assigned 570 every pixel a value on a gray scale of 0 (Black) - 255 (white), values of less than 50 correspond 571 to staining. We estimated a ratio by calculating the sum of these 50 pixels and dividing it by the 572 total number of pixels (150,000 pixels). Within each experiment, 14 animals from each condition 573 were quantified and all experiments were repeated at least three times on different days. Data 574 were analyzed for significance using Student's $t$ test and error bars represent SEM.

\section{Author Contributions}

577 H.E.L. conceived and conducted the experiments, interpreted the data, and co-wrote the paper.

578 Z.T.C wrote the tracker program, analyzed the tracking data, and co-wrote the paper. Z. L. and 579 C.J.Y. conducted behavioral assays. T.O.S. interpreted the data and provided guidance for the 580 data analysis. S.H.C. conceived the experiments, interpreted the data and co-wrote the paper.

\section{Acknowledgements}

583 We thank A. Dillin, T. Ishihara, S. Lockery, A. Samuelson, E. Troemel, M. Zhen, the National 584 BioResource Project (NBRP, Japan) and Caenorhabditis Genetics Center (CGC) for strains; C. 585 Bargmann, E. Hallem, M. Hilliard, A. van der Linden, P. McGrath, D. Pilgrim and P. Sengupta 586 for constructs; S. Srinivasan and lab members for RNAi clones and help with oil red O staining. 587 We are also grateful to L. Stowers, J. Wang, S. Asinof, L. Hale, U. Magaram, L. Shipp, K. 588 Quach, C. Yeh and members of the Chalasani lab for critical comments, advice, and insights. 
This work was funded by grants from The Rita Allen Foundation, The W.M. Keck Foundation and NIH R01MH096881-03 to S.H.C. H.E.L. $\square$ was initially supported by the Socrates Program funded by NSF GK-12 STEM Fellows in Education (Award \#NSF-742551) followed by a Graduate Research Fellowship also from the NSF.

\section{References}

Air, E.L., Benoit, S.C., Clegg, D.J., Seeley, R.J., and Woods, S.C. (2002). Insulin and leptin combine additively to reduce food intake and body weight in rats. Endocrinology 143, 2449-

5972452.

598 Akerboom, J., Chen, T.W., Wardill, T.J., Tian, L., Marvin, J.S., Mutlu, S., Calderon, N.C., Esposti, F., Borghuis, B.G., Sun, X.R., et al. (2012). Optimization of a GCaMP calcium indicator for neural activity imaging. J Neurosci 32, 13819-13840. M.H., Shanabrough, M., Cline, G., Shulman, G.I., et al. (2008). UCP2 mediates ghrelin's action on NPY/AgRP neurons by lowering free radicals. Nature 454, 846-851.

604 Atasoy, D., Betley, J.N., Su, H.H., and Sternson, S.M. (2012). Deconstruction of a neural circuit for hunger. Nature 488, 172-177. Avery, L. (1993). The genetics of feeding in Caenorhabditis elegans. Genetics 133, 897-917. Avery, L., and Horvitz, H.R. (1990). Effects of starvation and neuroactive drugs on feeding in Caenorhabditis elegans. J Exp Zool 253, 263-270. boss? Curr Opin Neurobiol 21, 888-896. Brenner, S. (1974). The genetics of Caenorhabditis elegans. Genetics 77, 71-94. $86,428-441$.

Carlini, V.P., Varas, M.M., Cragnolini, A.B., Schioth, H.B., Scimonelli, T.N., and de Barioglio, S.R. (2004). Differential role of the hippocampus, amygdala, and dorsal raphe nucleus in regulating feeding, memory, and anxiety-like behavioral responses to ghrelin. Biochem Biophys Res Commun 313, 635-641.

Carrillo, M.A., and Hallem, E.A. (2015). Gas sensing in nematodes. Mol Neurobiol 51, 919-931. Chalasani, S.H., Chronis, N., Tsunozaki, M., Gray, J.M., Ramot, D., Goodman, M.B., and Bargmann, C.I. (2007). Dissecting a circuit for olfactory behaviour in Caenorhabditis elegans. Nature 450, 63-70. (2010). Neuropeptide feedback modifies odor-evoked dynamics in Caenorhabditis elegans olfactory neurons. Nat Neurosci 13, 615-621.

Chen, A.T., Guo, C., Dumas, K.J., Ashrafi, K., and Hu, P.J. (2013a). Effects of Caenorhabditis elegans sgk-1 mutations on lifespan, stress resistance, and DAF-16/FoxO regulation. Aging Cell 12, 932-940.

Chen, Z., Hendricks, M., Cornils, A., Maier, W., Alcedo, J., and Zhang, Y. (2013b). Two insulin-like peptides antagonistically regulate aversive olfactory learning in C. elegans. Neuron $77,572-585$. 
632 Cowley, M.A., Smith, R.G., Diano, S., Tschop, M., Pronchuk, N., Grove, K.L., Strasburger, C.J.,

633 Bidlingmaier, M., Esterman, M., Heiman, M.L., et al. (2003). The distribution and mechanism of 634 action of ghrelin in the CNS demonstrates a novel hypothalamic circuit regulating energy 635 homeostasis. Neuron 37, 649-661.

636 de Bono, M., and Bargmann, C.I. (1998). Natural variation in a neuropeptide Y receptor homolog modifies social behavior and food response in C. elegans. Cell 94, 679-689.

638 Dietrich, M.O., and Horvath, T.L. (2012). Limitations in anti-obesity drug development: the critical role of hunger-promoting neurons. Nat Rev Drug Discov 11, 675-691.

640 Dietrich, M.O., and Horvath, T.L. (2013). Hypothalamic control of energy balance: insights into the role of synaptic plasticity. Trends Neurosci 36, 65-73. Dietrich, M.O., Zimmer, M.R., Bober, J., and Horvath, T.L. (2015). Hypothalamic Agrp neurons drive stereotypic behaviors beyond feeding. Cell 160, 1222-1232.

644 Doi, M., and Iwasaki, K. (2002). Regulation of retrograde signaling at neuromuscular junctions by the novel C2 domain protein AEX-1. Neuron 33, 249-259. specific knock-down of gene function in targeted C. elegans neurons. Gene 395, 170-176.

648 Ezcurra, M., Tanizawa, Y., Swoboda, P., and Schafer, W.R. (2011). Food sensitizes C. elegans avoidance behaviours through acute dopamine signalling. Embo J 30, 1110-1122. Figlewicz, D.P., and Sipols, A.J. (2010). Energy regulatory signals and food reward. Pharmacol Biochem Behav 97, 15-24.

652 Gallagher, T., Kim, J., Oldenbroek, M., Kerr, R., and You, Y.J. (2013). ASI regulates satiety quiescence in C. elegans. J Neurosci 33, 9716-9724.

654 Gillette, R., Huang, R.C., Hatcher, N., and Moroz, L.L. (2000). Cost-benefit analysis potential in feeding behavior of a predatory snail by integration of hunger, taste, and pain. Proc Natl Acad Sci U S A 97, 3585-3590.

657 Gray, J.M., Hill, J.J., and Bargmann, C.I. (2005). A circuit for navigation in Caenorhabditis elegans. Proc Natl Acad Sci U S A 102, 3184-3191. Walhout, A.J. (2009). A multiparameter network reveals extensive divergence between C. elegans bHLH transcription factors. Cell 138, 314-327.

662 Gruninger, T.R., Gualberto, D.G., and Garcia, L.R. (2008). Sensory perception of food and insulin-like signals influence seizure susceptibility. PLoS Genet 4, e1000117.

664 Hamm, H.E. (1998). The many faces of G protein signaling. J Biol Chem 273, 669-672. Havula, E., and Hietakangas, V. (2012). Glucose sensing by ChREBP/MondoA-Mlx transcription factors. Semin Cell Dev Biol 23, 640-647. in the Akt/PKB kinase complex to control stress response and life span. Dev Cell 6, 577-588. In vivo imaging of $\mathrm{C}$. elegans $\mathrm{ASH}$ neurons: cellular response and adaptation to chemical repellents. Embo J 24, 63-72.

673 Hilliard, M.A., and Bargmann, C.I. (2006). Wnt signals and frizzled activity orient anteriorposterior axon outgrowth in C. elegans. Dev Cell 10, 379-390. Hilliard, M.A., Bargmann, C.I., and Bazzicalupo, P. (2002). C. elegans responds to chemical repellents by integrating sensory inputs from the head and the tail. Curr Biol 12, 730-734. search behavior in Caenorhabditis elegans. J Neurosci 24, 1217-1225. 
Hobert, O. (2013). The neuronal genome of Caenorhabditis elegans. WormBook, 1-106. Hung, W.L., Wang, Y., Chitturi, J., and Zhen, M. (2014). A Caenorhabditis elegans developmental decision requires insulin signaling-mediated neuron-intestine communication. Development 141, 1767-1779. Husson, S.J., Janssen, T., Baggerman, G., Bogert, B., Kahn-Kirby, A.H., Ashrafi, K., and Schoofs, L. (2007). Impaired processing of FLP and NLP peptides in carboxypeptidase E (EGL21)-deficient Caenorhabditis elegans as analyzed by mass spectrometry. J Neurochem 102, 246260. Iino, Y., and Yoshida, K. (2009). Parallel use of two behavioral mechanisms for chemotaxis in Caenorhabditis elegans. J Neurosci 29, 5370-5380. Inagaki, H.K., Panse, K.M., and Anderson, D.J. (2014). Independent, reciprocal neuromodulatory control of sweet and bitter taste sensitivity during starvation in Drosophila. Neuron 84, 806-820. Ishihara, T., Iino, Y., Mohri, A., Mori, I., Gengyo-Ando, K., Mitani, S., and Katsura, I. (2002). HEN-1, a secretory protein with an LDL receptor motif, regulates sensory integration and learning in Caenorhabditis elegans. Cell 109, 639-649.

Jansen, G., Thijssen, K.L., Werner, P., van der Horst, M., Hazendonk, E., and Plasterk, R.H. (1999). The complete family of genes encoding G proteins of Caenorhabditis elegans. Nat Genet 21, 414-419.

Johnson, D.W., Llop, J.R., Farrell, S.F., Yuan, J., Stolzenburg, L.R., and Samuelson, A.V. (2014). The Caenorhabditis elegans Myc-Mondo/Mad complexes integrate diverse longevity signals. PLoS Genet 10, e1004278. Jones, K.T., Greer, E.R., Pearce, D., and Ashrafi, K. (2009). Rictor/TORC2 regulates Caenorhabditis elegans fat storage, body size, and development through sgk-1. PLoS Biol 7, e60. Jordan, S.D., Konner, A.C., and Bruning, J.C. (2010). Sensing the fuels: glucose and lipid signaling in the CNS controlling energy homeostasis. Cell Mol Life Sci 67, 3255-3273. Kawai, K., Sugimoto, K., Nakashima, K., Miura, H., and Ninomiya, Y. (2000). Leptin as a modulator of sweet taste sensitivities in mice. Proc Natl Acad Sci U S A 97, 11044-11049.

Kojima, M., Hosoda, H., Date, Y., Nakazato, M., Matsuo, H., and Kangawa, K. (1999). Ghrelin is a growth-hormone-releasing acylated peptide from stomach. Nature 402, 656-660. Lapierre, L.R., and Hansen, M. (2012). Lessons from C. elegans: signaling pathways for longevity. Trends Endocrinol Metab 23, 637-644.

Leinwand, S.G., and Chalasani, S.H. (2013). Neuropeptide signaling remodels chemosensory circuit composition in Caenorhabditis elegans. Nat Neurosci 16, 1461-1467.

Li, C., and Kim, K. (2008). Neuropeptides. WormBook, 1-36.

Li, M.V., Chen, W., Harmancey, R.N., Nuotio-Antar, A.M., Imamura, M., Saha, P., Taegtmeyer, H., and Chan, L. (2010). Glucose-6-phosphate mediates activation of the carbohydrate responsive binding protein (ChREBP). Biochem Biophys Res Commun 395, 395-400.

Lipton, J., Kleemann, G., Ghosh, R., Lints, R., and Emmons, S.W. (2004). Mate searching in 718 7427-7434. Caenorhabditis elegans: a genetic model for sex drive in a simple invertebrate. J Neurosci 24 ,

Ludewig, A.H., and Schroeder, F.C. (2013). Ascaroside signaling in C. elegans. WormBook, 122.

Maduro, M., and Pilgrim, D. (1995). Identification and cloning of unc-119, a gene expressed in the Caenorhabditis elegans nervous system. Genetics 141, 977-988. 
Mahoney, T.R., Luo, S., Round, E.K., Brauner, M., Gottschalk, A., Thomas, J.H., and Nonet, M.L. (2008). Intestinal signaling to GABAergic neurons regulates a rhythmic behavior in Caenorhabditis elegans. Proc Natl Acad Sci U S A 105, 16350-16355. Malik, S., McGlone, F., Bedrossian, D., and Dagher, A. (2008). Ghrelin modulates brain activity in areas that control appetitive behavior. Cell Metab 7, 400-409. McGhee, J.D. (2007). The C. elegans intestine. WormBook, 1-36. McGlone, F., and Reilly, D. (2010). The cutaneous sensory system. Neurosci Biobehav Rev 34, $730 \quad 148-159$. Mease, R.A., Famulare, M., Gjorgjieva, J., Moody, W.J., and Fairhall, A.L. (2013). Emergence of adaptive computation by single neurons in the developing cortex. J Neurosci 33, 1215412170.

Mercer, R.E., Chee, M.J., and Colmers, W.F. (2011). The role of NPY in hypothalamic mediated food intake. Front Neuroendocrinol 32, 398-415.

Mizunuma, M., Neumann-Haefelin, E., Moroz, N., Li, Y., and Blackwell, T.K. (2014). mTORC2-SGK-1 acts in two environmentally responsive pathways with opposing effects on longevity. Aging Cell 13, 869-878.

Moerman, D.G., and Williams, B.D. (2006). Sarcomere assembly in C. elegans muscle. WormBook, 1-16.

Muller, T.D., Nogueiras, R., Andermann, M.L., Andrews, Z.B., Anker, S.D., Argente, J., Batterham, R.L., Benoit, S.C., Bowers, C.Y., Broglio, F., et al. (2015). Ghrelin. Molecular metabolism 4, 437-460.

Murphy, C.T., and Hu, P.J. (2013). Insulin/insulin-like growth factor signaling in C. elegans. WormBook, 1-43.

Nassel, D.R., and Wegener, C. (2011). A comparative review of short and long neuropeptide F signaling in invertebrates: Any similarities to vertebrate neuropeptide Y signaling? Peptides 32, 1335-1355. requirements for myosin gene expression and regulation in Caenorhabditis elegans. Genetics 135, 385-404.

Pierce, S.B., Costa, M., Wisotzkey, R., Devadhar, S., Homburger, S.A., Buchman, A.R., Ferguson, K.C., Heller, J., Platt, D.M., Pasquinelli, A.A., et al. (2001). Regulation of DAF-2 receptor signaling by human insulin and ins-1, a member of the unusually large and diverse $\mathrm{C}$. elegans insulin gene family. Genes Dev 15, 672-686.

Pierce-Shimomura, J.T., Morse, T.M., and Lockery, S.R. (1999). The fundamental role of pirouettes in Caenorhabditis elegans chemotaxis. J Neurosci 19, 9557-9569.

Sawin, E.R., Ranganathan, R., and Horvitz, H.R. (2000). C. elegans locomotory rate is modulated by the environment through a dopaminergic pathway and by experience through a serotonergic pathway. Neuron 26, 619-631.

Schrodel, T., Prevedel, R., Aumayr, K., Zimmer, M., and Vaziri, A. (2013). Brain-wide 3D imaging of neuronal activity in Caenorhabditis elegans with sculpted light. Nat Methods 10, 1013-1020.

764 Sengupta, P. (2013). The belly rules the nose: feeding state-dependent modulation of peripheral chemosensory responses. Curr Opin Neurobiol 23, 68-75.

Sheng, M., Hosseinzadeh, A., Muralidharan, S.V., Gaur, R., Selstam, E., and Tuck, S. (2015). program. PLoS One 10, e0124515. 
Shioi, G., Shoji, M., Nakamura, M., Ishihara, T., Katsura, I., Fujisawa, H., and Takagi, S. (2001). Mutations affecting nerve attachment of Caenorhabditis elegans. Genetics 157, 1611-1622. Soukas, A.A., Kane, E.A., Carr, C.E., Melo, J.A., and Ruvkun, G. (2009). Rictor/TORC2 regulates fat metabolism, feeding, growth, and life span in Caenorhabditis elegans. Genes Dev 23, 496-511. Martin, T.F. (2007). UNC-31 (CAPS) is required for dense-core vesicle but not synaptic vesicle exocytosis in Caenorhabditis elegans. J Neurosci 27, 6150-6162. Speidel, D., Varoqueaux, F., Enk, C., Nojiri, M., Grishanin, R.N., Martin, T.F., Hofmann, K., Brose, N., and Reim, K. (2003). A family of Ca2+-dependent activator proteins for secretion: comparative analysis of structure, expression, localization, and function. J Biol Chem 278, 52802-52809. processes for hunger. Curr Opin Neurobiol 23, 353-360. the carbohydrate response element-binding protein in glucose regulation of lipogenic enzyme genes. J Biol Chem 279, 15662-15669. Glucose sensing by MondoA:Mlx complexes: a role for hexokinases and direct regulation of thioredoxin-interacting protein expression. Proc Natl Acad Sci U S A 105, 6912-6917.

Taghert, P.H., and Nitabach, M.N. (2012). Peptide neuromodulation in invertebrate model systems. Neuron 76, 82-97.

Trent, C., Tsuing, N., and Horvitz, H.R. (1983). Egg-laying defective mutants of the nematode Caenorhabditis elegans. Genetics 104, 619-647.

Tschop, M., Smiley, D.L., and Heiman, M.L. (2000). Ghrelin induces adiposity in rodents. Nature 407, 908-913.

Wark, B., Fairhall, A., and Rieke, F. (2009). Timescales of inference in visual adaptation. Neuron 61, 750-761.

Wark, B., Lundstrom, B.N., and Fairhall, A. (2007). Sensory adaptation. Curr Opin Neurobiol $17,423-429$.

800

Warren, C.E., Krizus, A., and Dennis, J.W. (2001). Complementary expression patterns of six

801

802

803

804 nonessential Caenorhabditis elegans core 2/I N-acetylglucosaminyltransferase homologues. Glycobiology 11, 979-988.

White, J.G., Southgate, E., Thomson, J.N., and Brenner, S. (1986). The structure of the nervous system of the nematode Caenorhabditis elegans. Philos Trans R Soc Lond B Biol Sci 314, 1-340.

807 Figure 1. Food deprivation specifically and reversibly alters repellent-driven behaviors. (A)

808 Schematic of sensory integration assay with the copper barrier (blue) and animals at the origin

809 and the attractant on the other side. (B) Food-deprived animals show increased integration index

810 compared to well-fed animals integrating 50mM copper sulfate with 1:500 diacetyl. Two-tailed

811 Student's $t$ test $* * * \mathrm{p}<0.0001, \mathrm{n} \geq 9$. (C) Compared to well-fed animals, food-deprived animals 
812 cross the copper sulfate barrier significantly more even when no attractant is presented in the

813 assay. Chemotaxis to diacetyl alone is not modified by food deprivation. One sample $t$ test $* * * p$

$814<0.0001, \mathrm{n} \geq 9$ per condition per assay. (D) Time course of food deprivation shows that a

815 minimum two hours of food deprivation is required for modifying behavior. Maximum

816 behavioral modification can be observed after three hours of food deprivation. One-way

817 ANOVA $* * * \mathrm{p}<0.0001, \mathrm{n} \geq 9$ per condition per time point. (E) After three hours of food

818 deprivation, five hours of recovery on food reverts sensory integration behavior to well-fed

819 levels. One-way ANOVA $* * * \mathrm{p}<0.0001, \mathrm{n} \geq 9$ per condition per time point. (F) Animals

820 exposed to aztreonam-treated $E$. coli for three hours show similar reduction in copper sensitivity

821 as food-deprived animals. One sample $t$ test $\mathrm{p}<0.05, \mathrm{n} \geq 9$ per condition. All bars represent

822 population means; error bars indicate SEM. See also Figure S1-S2, S7.

823

824 Figure 2. Food deprivation reduces reorientation events enabling increased repellent

825 barrier crossing. (A-B) Tracks of (A) well-fed and (B) food-deprived animals in sensory 826 integration assay over 45 minutes. Blue line in the middle indicates the position of copper 827 barrier. Animals begin $-10 \mathrm{~mm}$ from copper barrier and diacetyl is positioned at $10 \mathrm{~mm}$ from 828 copper barrier at the orange spot. ( $n=48$ for each condition). (C) Reorientation probability of 829 well-fed and food-deprived animals moving towards (left half) and away (right half) from the 830 copper line during sensory integration assay. Well-fed animals exhibit increased reorientation 831 probability approaching copper line. (D) Small-turn probability of well-fed and food-deprived 832 animals moving towards (left half) and away (right half) from the copper line during sensory 833 integration assay. Well-fed and food-deprived animals exhibit similar small-turn behaviors. All 834 data points represent median of bootstrap reorientation probability estimates; error bars indicate $83550 \%$ bootstrap bias-corrected confidence interval $(\mathrm{n}>100$, bootstraps $=1000, * 95 \% \mathrm{CI})$. See 836 also Figure S3.

838 Figure 3. Multiple tissues sense lack of food with MML-1, process and release 839 neuropeptides to modify behavior upon food deprivation. (A) $m m l-1$, but not $m x l-2$ mutants 840 are defective in modifying integration responses after food deprivation. Restoring $m m l-1$ cDNA 841 in the intestine or body wall muscles but not neurons is sufficient to restore normal food 842 deprivation behavior to $m m l-1$ mutants. (B) Schematic representation of promoters used for 
843 tissue-specific expression. Pan-neuronal expression is achieved using unc-119 or H20 promoter,

844 intestine expression using gly-19 promoter, body wall muscle expression using myo-3 promoter,

845 pharyngeal muscle expression using myo-2 promoter, and tail hypodermal cell expression using

846 lin-44 promoter. (C) Schematic representation of proteins required for neuropeptide processing 847 and release. Pro-peptides are cleaved by pro-protein convertases (AEX-5, EGL-3, BLI-4 and 848 KPC-1) and further processed before they are packed into dense core vesicles and released using 849 UNC-31 (CAPS protein). (D) AEX-5 and EGL-3, but not BLI-4 or KPC-1 processed peptides 850 are required for sensory integration change after food deprivation. (E) Tissue-specific rescues of 851 aex-5 in either intestine, body wall muscles or neurons reverts aex-5 null mutants to normal 852 sensory integration response after food deprivation. (F) Tissue-specific knockdown of aex-5 in 853 wild-type animals in intestine, body wall muscles or neurons resulted in animals that were 854 defective in integration response after food deprivation. (G) Tissue-specific knockdown of unc85531 in the intestine, body wall muscles or neurons of wild-type animals does not modify 856 integration behavior after food deprivation, supporting a requirement for neuropeptide release 857 from these tissues. All bars represent population means; error bars indicate SEM. Unpaired $t$ test 858 with Welch's correction $* \mathrm{p}<0.05 ; * * \mathrm{p}<0.001 ; * * * \mathrm{p}<0.0001, \mathrm{n} \geq 9$ per condition per strain.

Figure 4. ASI chemosensory neurons use DAF-2 receptors to integrate intestine-released

861 neuropeptides. (A) daf-2 mutants are defective in their sensory integration response after food

862 deprivation. Rescue of $d a f-2$ in neurons, but not intestine or body wall muscles, is sufficient to 863 restore wild-type food deprivation behavior. (B) Restoring DAF-2 to ASI, but not ASH or ADL 864 neurons, is sufficient to partially restore integration response after food deprivation to daf-2 865 mutants. (C) Schematic diagram of candidate pathway components downstream of DAF-2 866 activation. Components in green are required for behavioral modification upon food deprivation.

867 (D) Animals with mutations in the $s g k-1$ and rict- 1 genes are defective in integration response to 868 food deprivation. Restoring $s g k-1$ and rict-1 to ASI neurons is sufficient to restore food869 deprivation induced behavioral modification in sgk-1 and rict-1 mutants, respectively. (E) 870 Rescue of aex-5 in the intestine and daf-2 in ASI neurons of aex-5;daf-2 double mutants is 871 sufficient to restore sensory integration response after food deprivation. Unpaired $t$ test with 872 Welch's correction $* \mathrm{p}<0.05 ; * * \mathrm{p}<0.001 ; * * * \mathrm{p}<0.0001, \mathrm{n} \geq 9$ per condition per strain. See 873 also Figure S4. 
875 Figure 5. Food deprivation alters ASI neuronal adaptation rate. (A) Responses of ASI neurons to 1 -second pulses of 50mM copper solution in wild-type well-fed (WF) and fooddeprived (FD) animals. Gray bars (16) represent stimulus pulses. Food-deprived animals do not readily adapt to repeated repellent pulses. Solid lines represent averaged traces, shading around each line indicate SEM ( $\mathrm{n} \geq 21)$. (B) Bar graph of averaged fluorescence over a 8s-window before and after peak response (red arrow in (A) indicates peak). Well-fed animals respond to copper with an increase leading to the peak response followed by decay after the peak. In contrast, ASI responses in food-deprived animals continue to rise to subsequent pulses. Error bar indicates SEM (Unpaired $t$ test with Welch's correction, *p $<0.05$, **p $<0.001, \mathrm{n} \geq 21$ ). (C)

884 Responses of ASH neurons to 1-second pulses of 50mM copper solution in well-fed and food885 deprived animals. Solid lines represent averaged traces, shading around each line indicate SEM 886 ( $\mathrm{n} \geq 21)$. (D) Bar graph of averaged fluorescence over a 8s-window before and after peak 887 response (red arrow in (C) indicates peak). ASH neurons in well-fed and food-deprived animals 888 respond similarly to copper before and after the peak. Error bar indicates SEM (Unpaired $t$ test with Welch's correction, *p <0.05, $\mathrm{n} \geq 21)$. See also Figure S5 - S6.

Figure 6. Modification of ASI neuronal adaptation rate after food deprivation requires DAF-2 signaling. (A) Responses of ASI neurons to 1-second pulses of $50 \mathrm{mM}$ copper solution in daf-2 mutant animals under well-fed (WF) and food-deprived (FD) conditions. Gray bars (16) 894 represent stimulus pulses. WF and FD daf-2 animals respond similarly to copper stimuli. Solid 895 lines represent averaged traces, shading around each line indicate SEM ( $\mathrm{n} \geq 13)$. (B) Bar graph 896 of averaged fluorescence over a 8s-window before and after peak response (red arrow in (A) 897 indicates peak). Well-fed and food-deprived daf-2 mutants respond to copper with an increase 898 leading to the peak response followed by decrease after the peak. Error bar indicates SEM 899 (Unpaired $t$ test with Welch's correction, *p < 0.05, $\mathrm{n} \geq 13$ ). (C) Responses of ASH neurons to 1900 second pulses of 50mM copper solution in well-fed and food-deprived daf-2 animals. Solid lines 901 represent averaged traces, shading around each line indicate SEM ( $n \geq 13)$. (D) Bar graph of 902 averaged fluorescence over a 8s-window before and after peak response (red arrow in (C) 903 indicates peak). ASH neurons in well-fed and food-deprived animals respond similarly to copper 904 before and after the peak. Error bar indicates SEM (Unpaired $t$ test with Welch's correction, *p < 
$9050.05, \mathrm{n} \geq 13$ ). (E) Percent increase of post-peak response compared to pre-peak response. Wild-

906 type food-deprived ASI, but not ASH neurons show a significant increase in their response to

907 copper stimuli even as the responses decline in well-fed condition. Moreover, these food

908 deprivation effects on ASI neurons are lost in daf-2 mutants. (F) Model for food-deprivation

909 induced modification of copper sensitivity. See also Figure S5 -S6.

910

911

912 
A

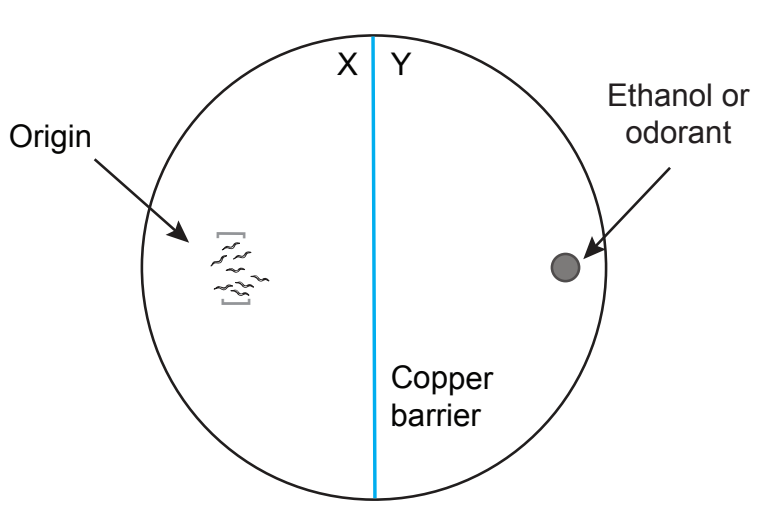

Integration index $=$ Number of animals in $Y$

Total number of animals

D

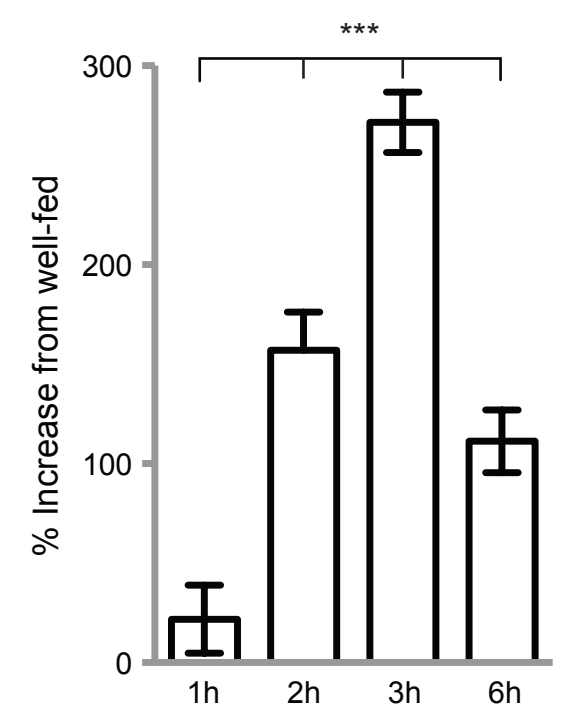

Hours of food deprivation
B

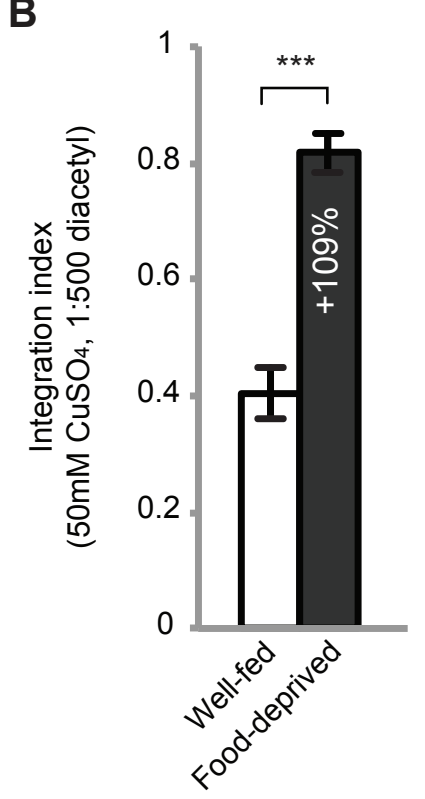

E

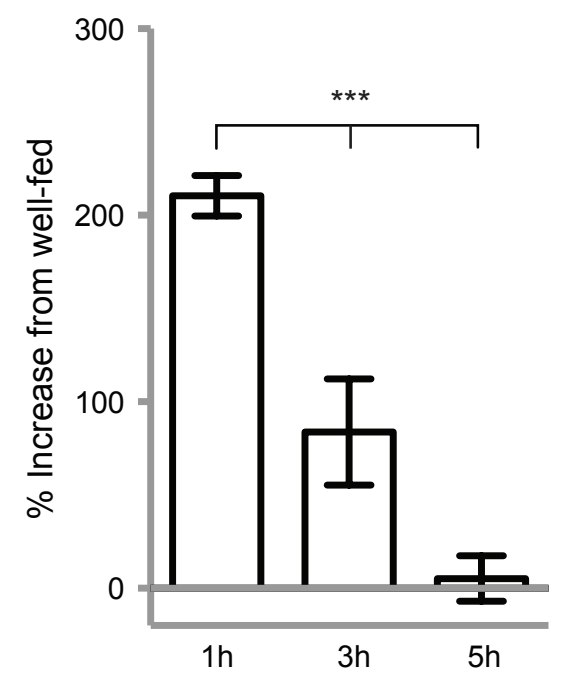

Hours of recovery on food after $3 \mathrm{~h}$ food deprivation
C

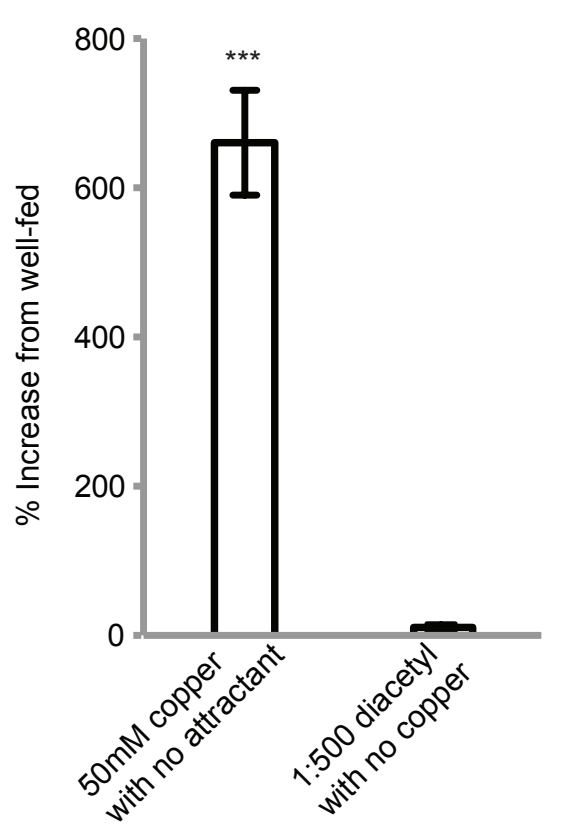

F

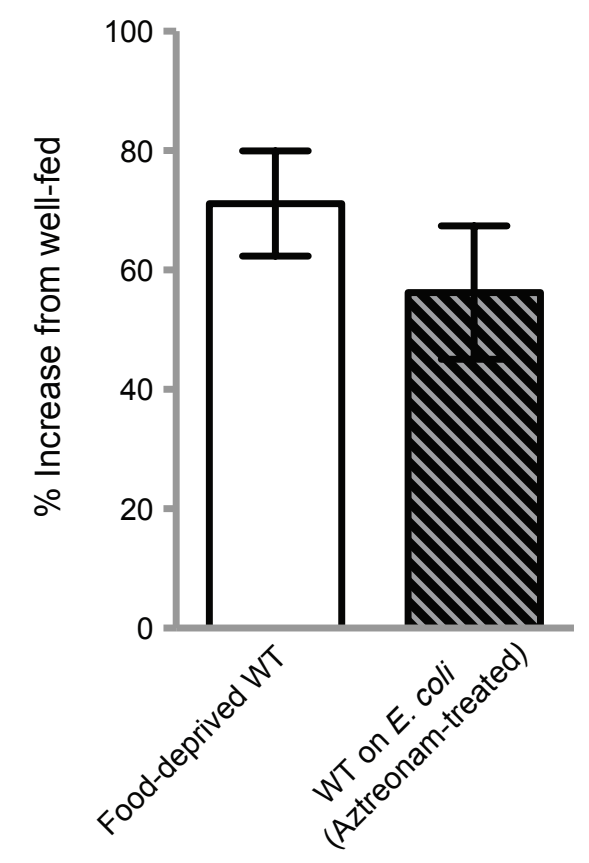


A

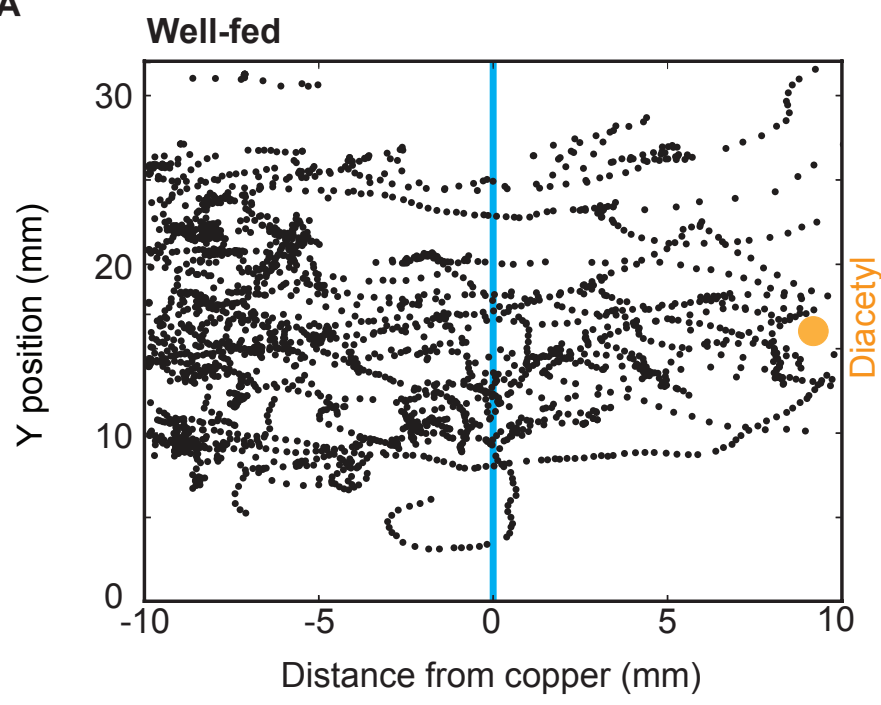

C

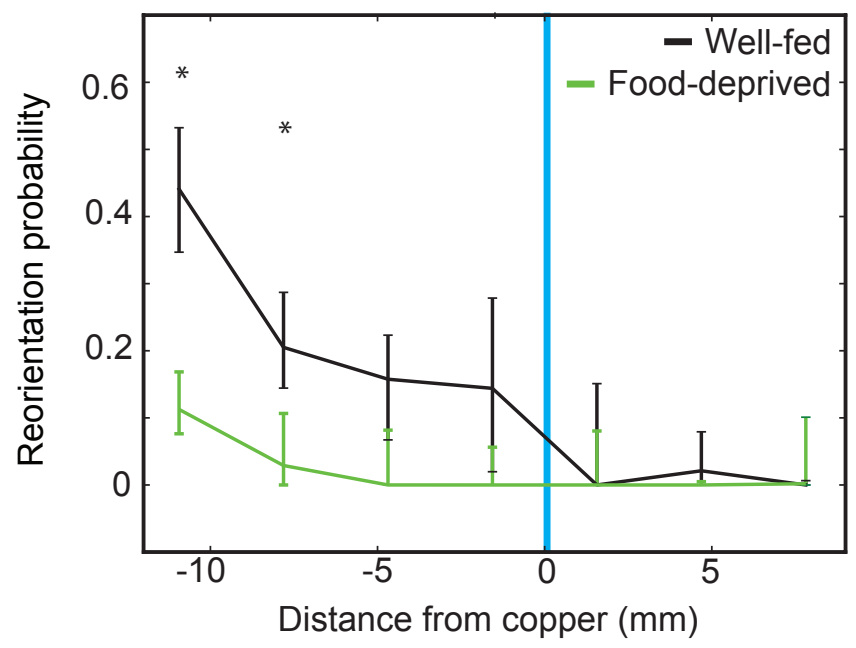

B

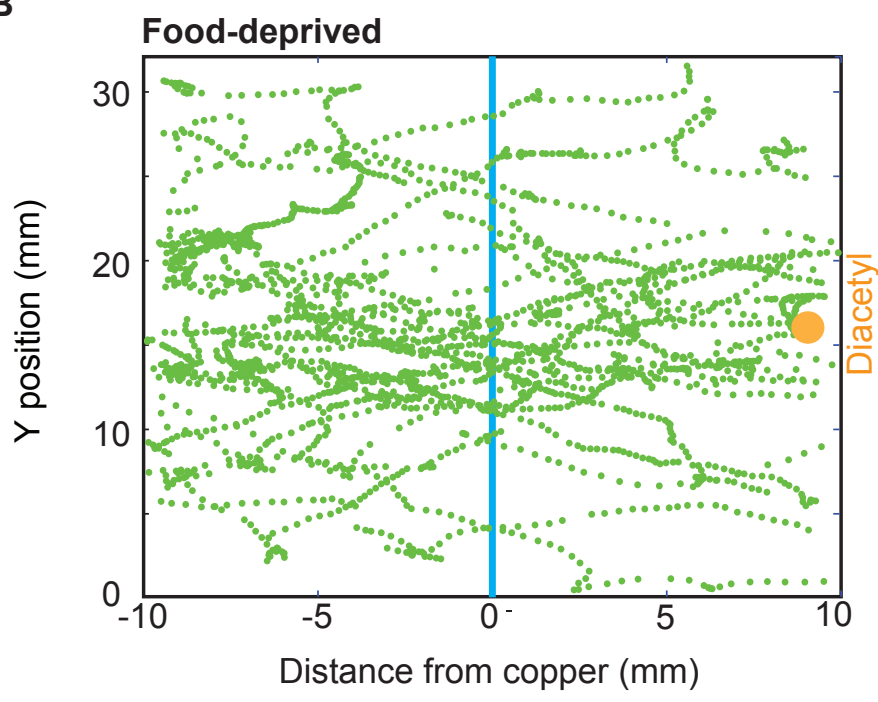

D

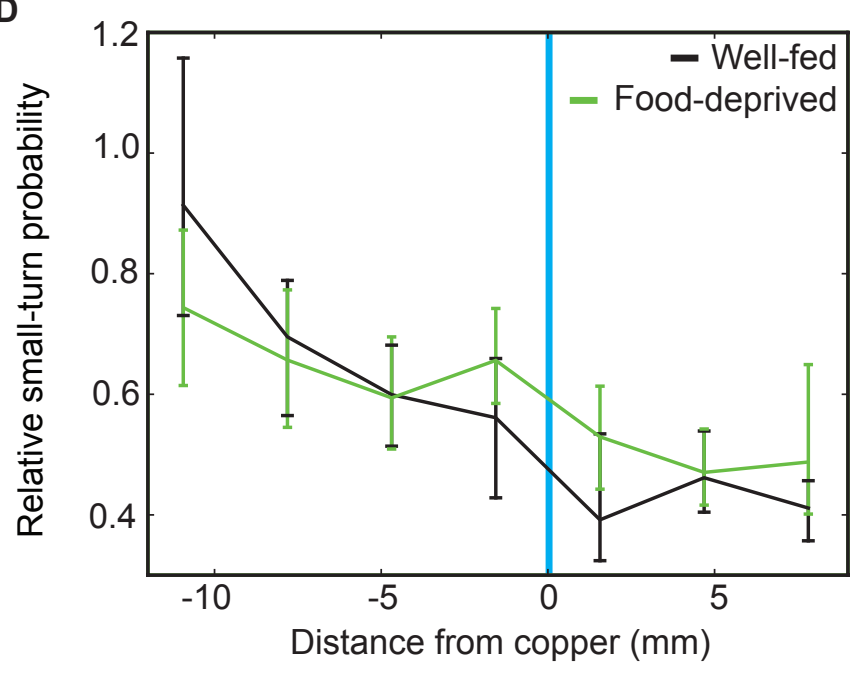


A

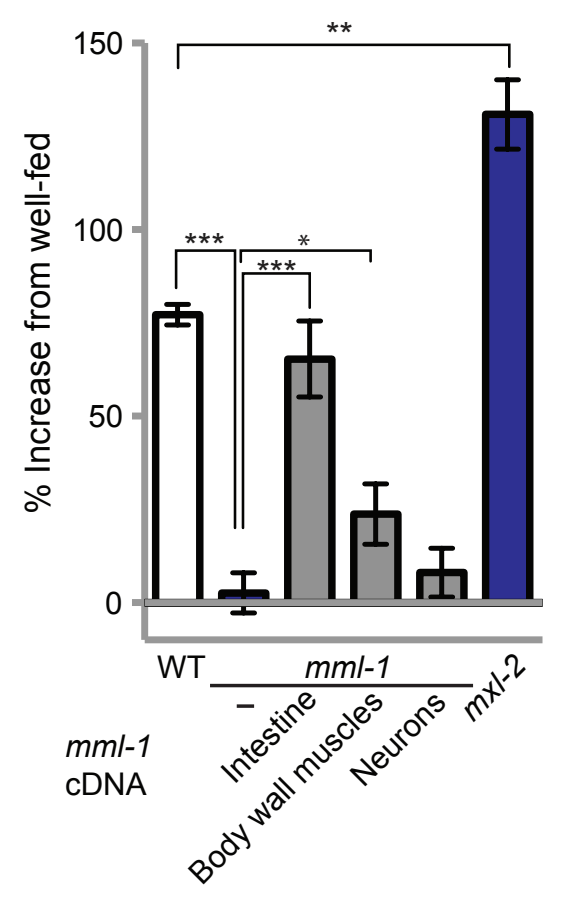

B

Tissue (promoter):

\section{All neurons}

(unc-119/H20)

Intestine

(gly-19)

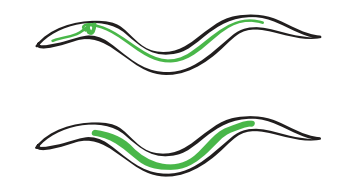

Body wall muscles

(myo-3)

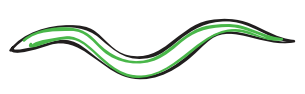

Pharynx

(myo-2)

Tail hypodemal cells

(lin-44)
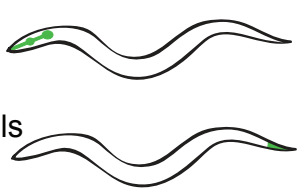

C

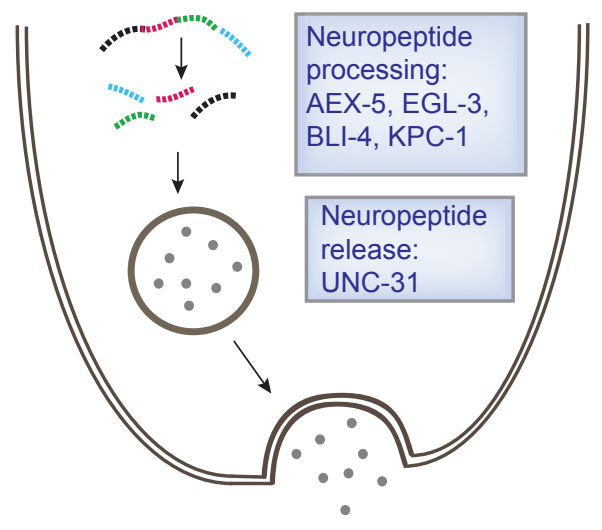

G
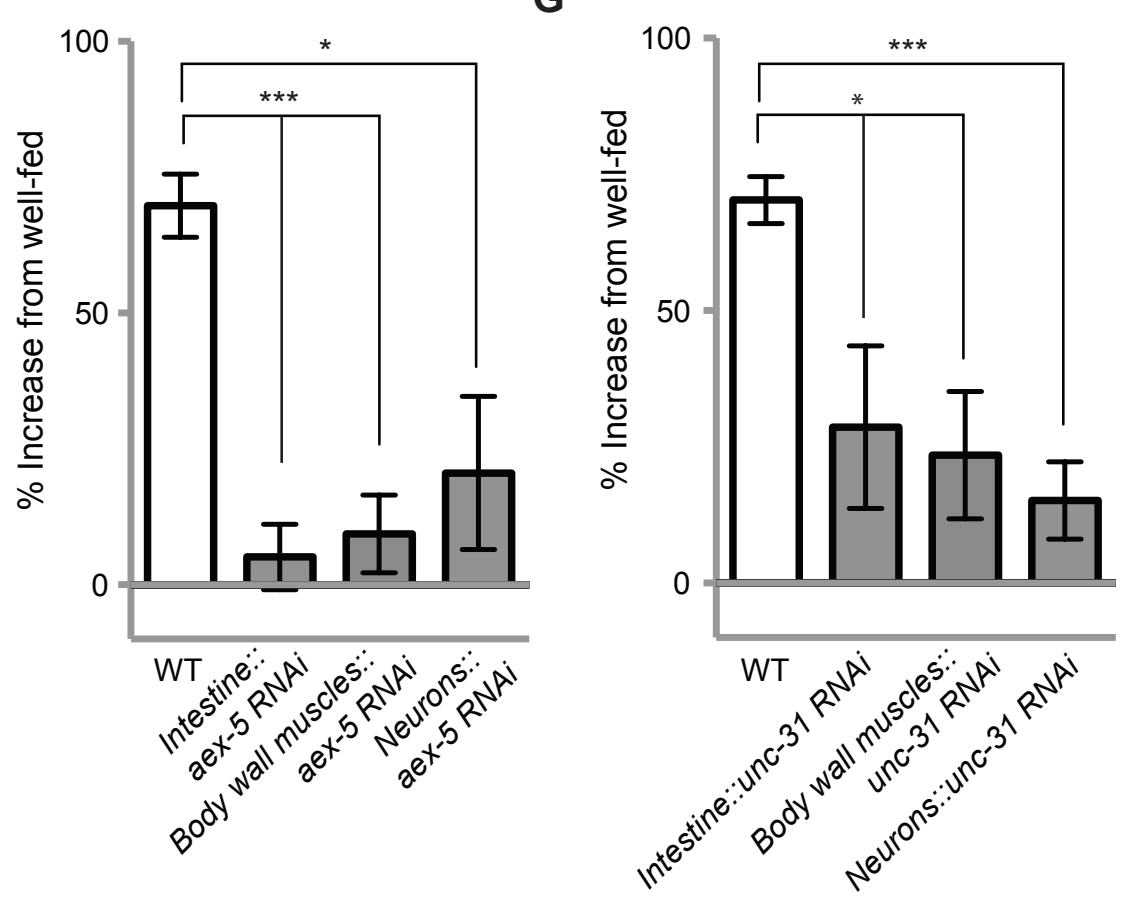

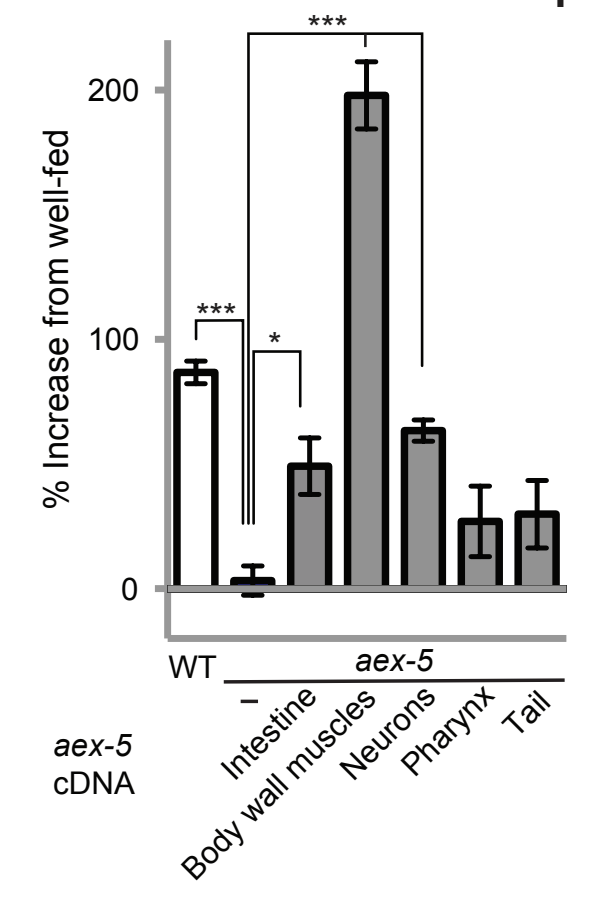


A

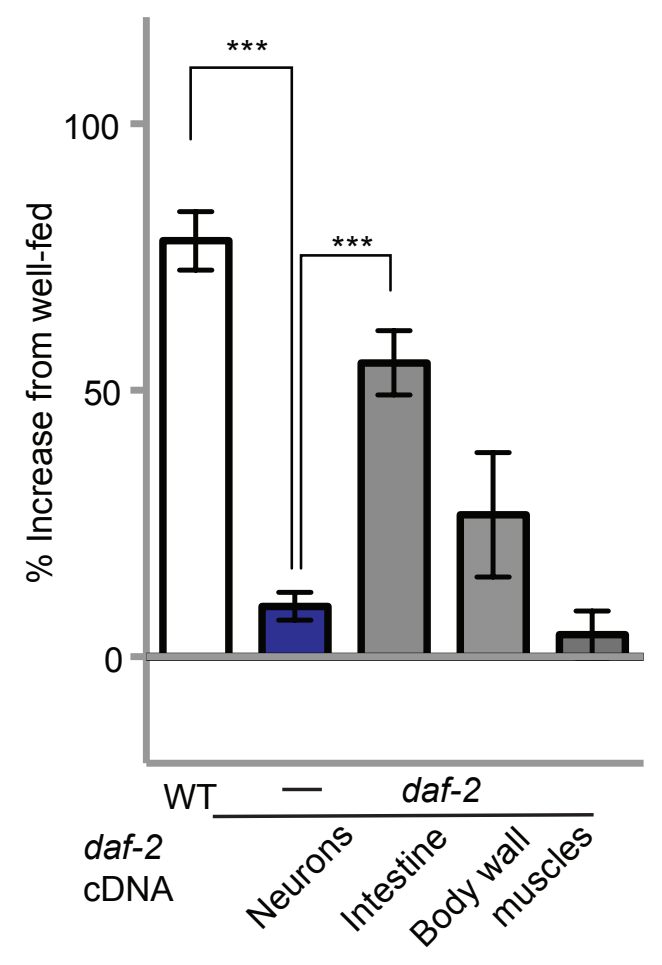

B

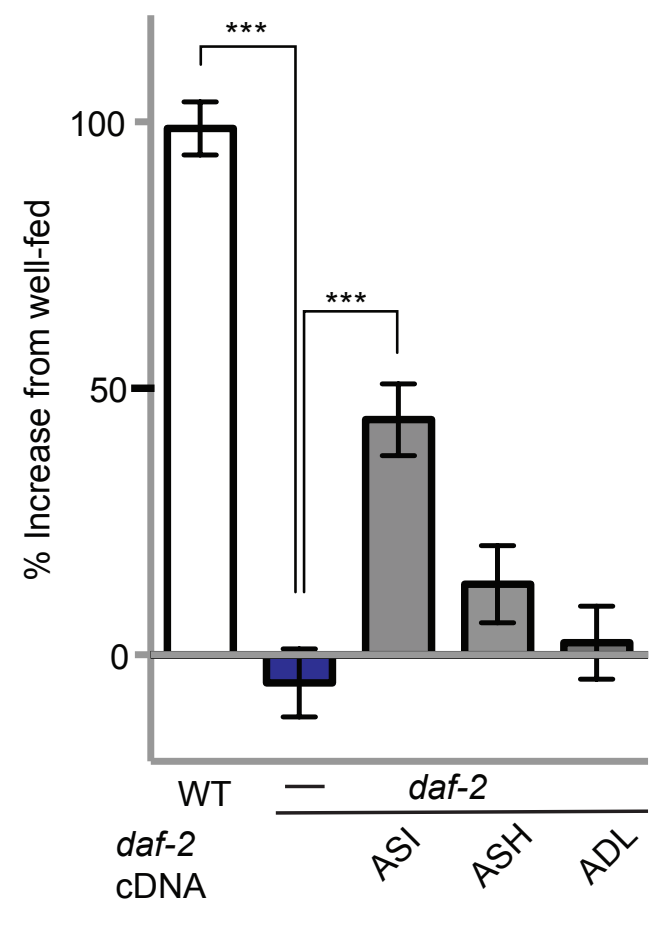

C

Neuropeptide(s)

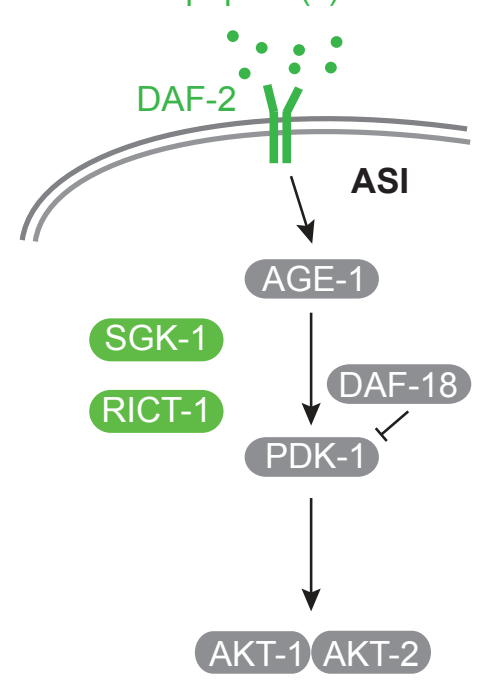

D

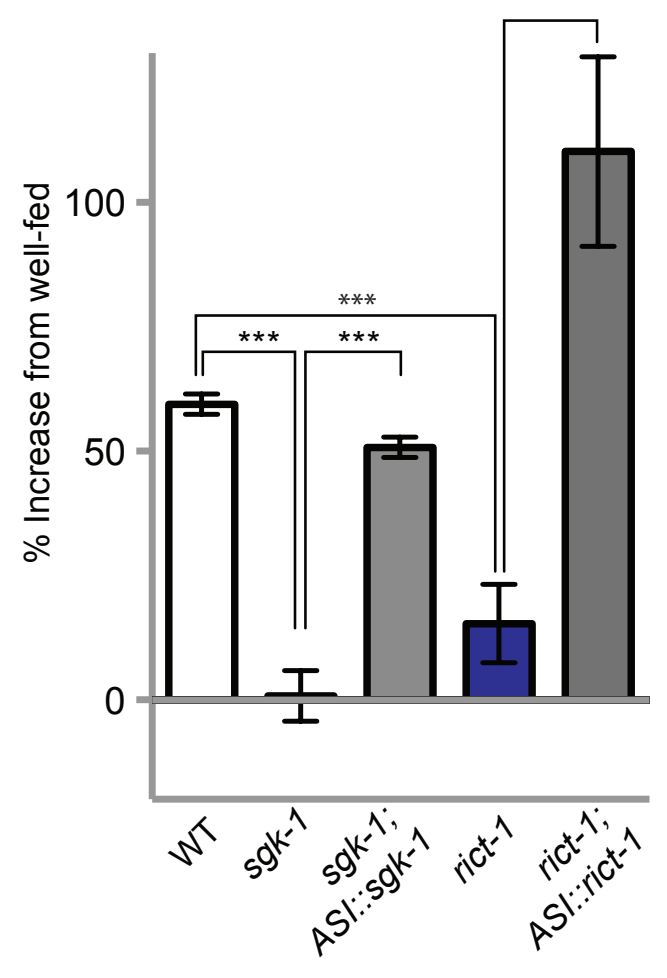

E

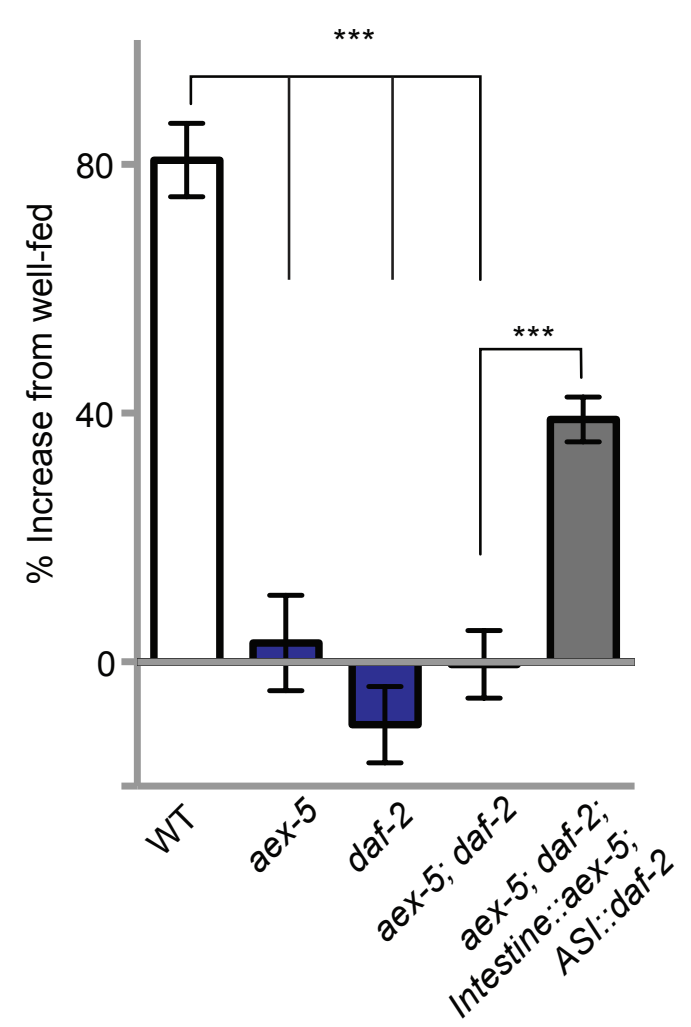


A

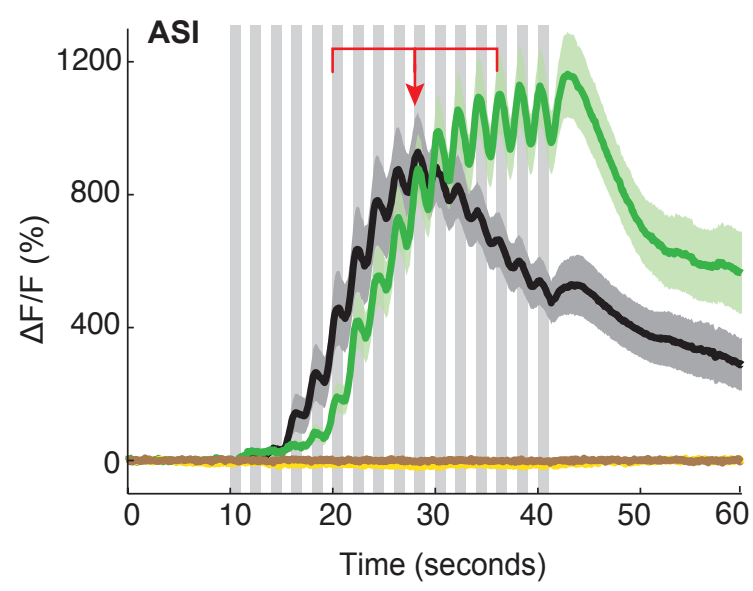

C

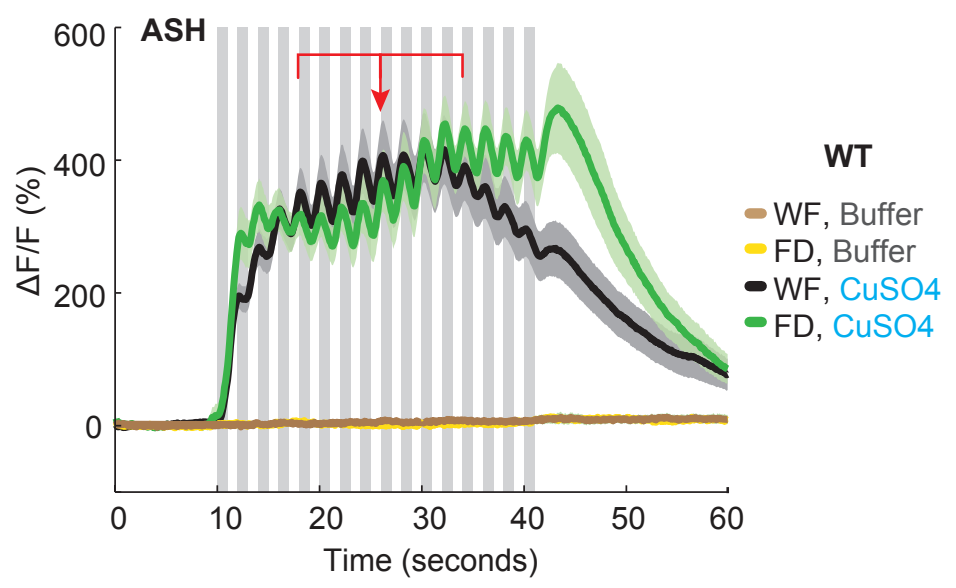

B

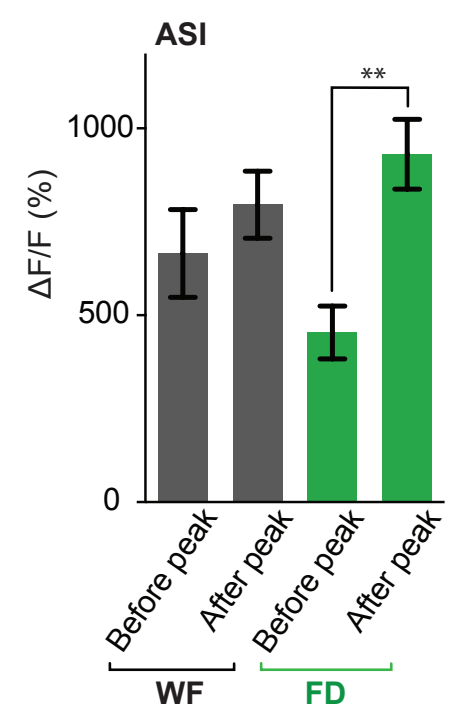

D

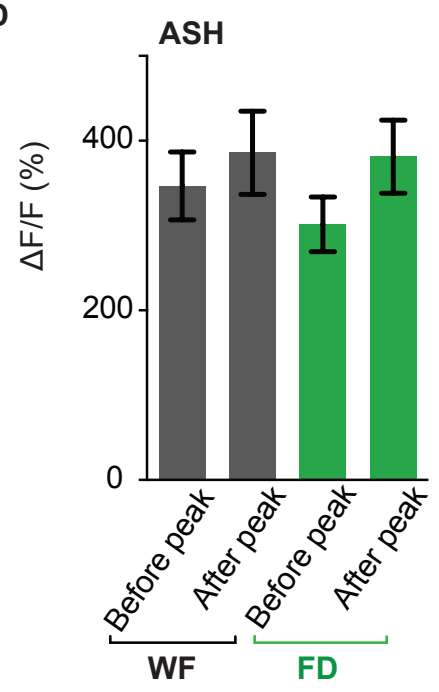

Lau_Figure5 
A

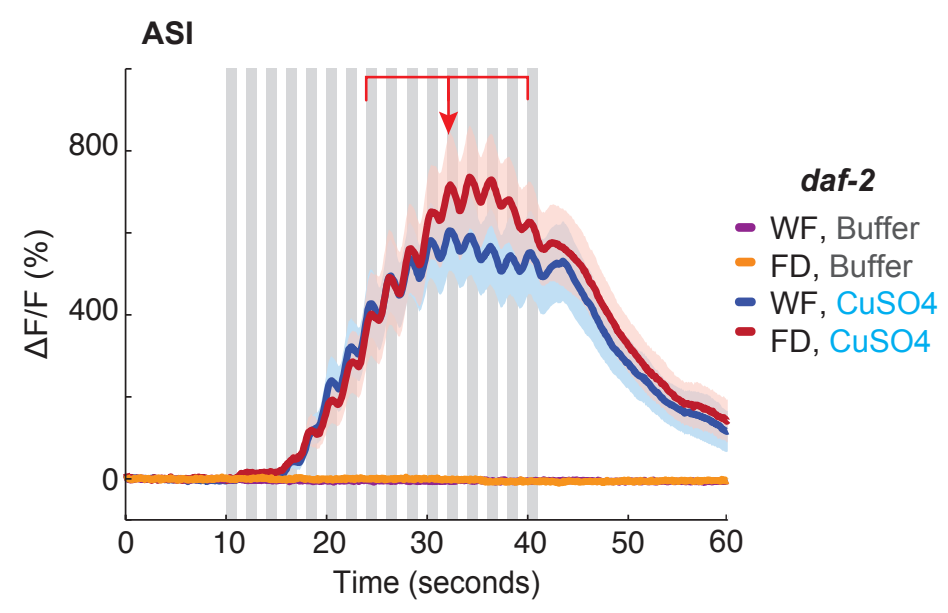

C

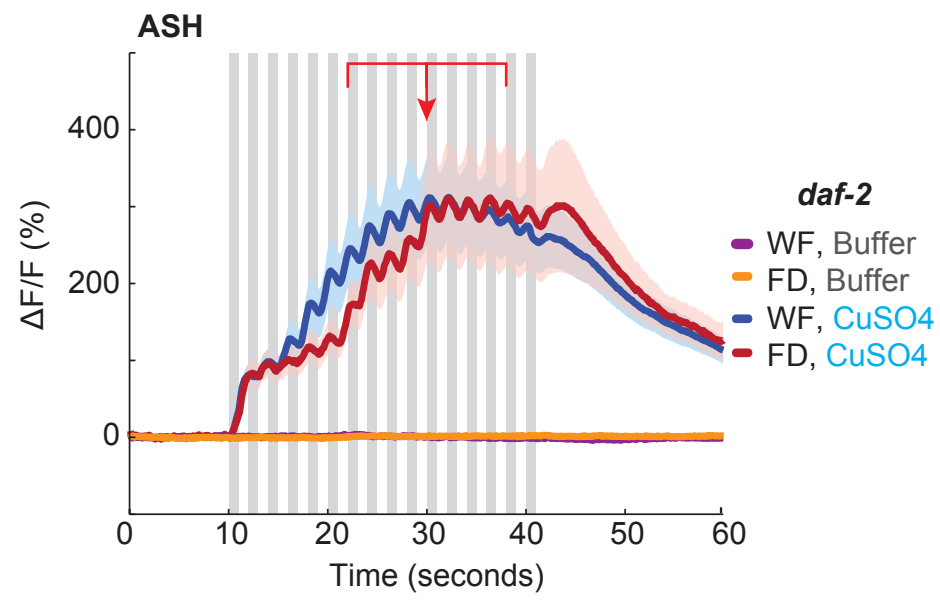

E

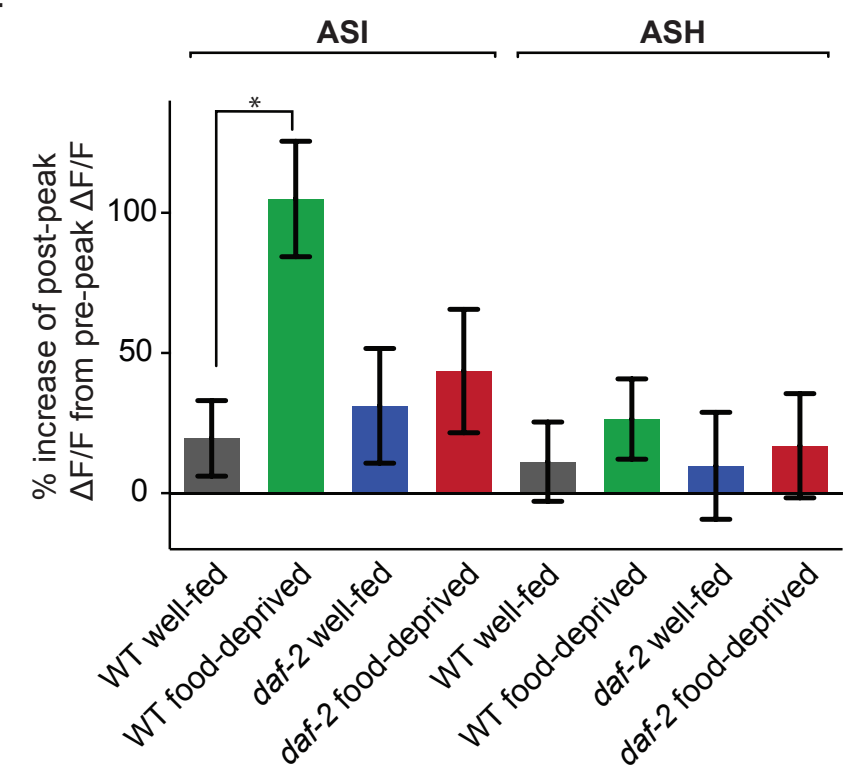

B

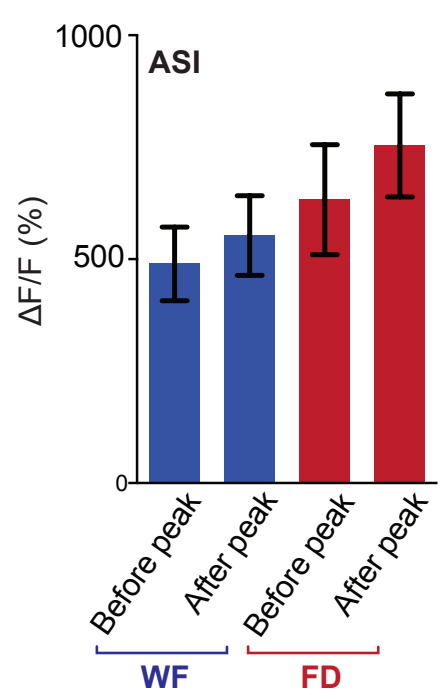

D

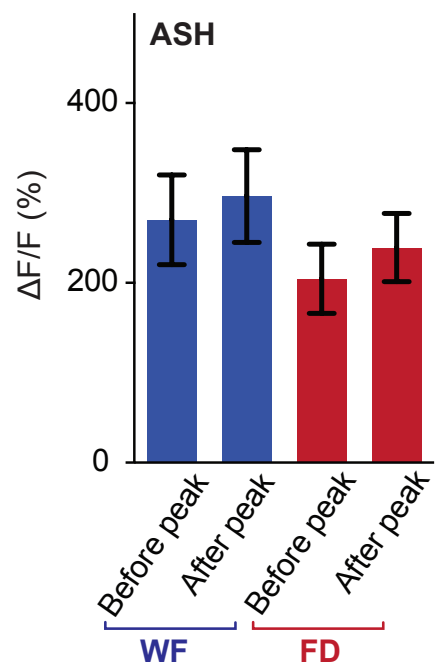

$\mathbf{F}$

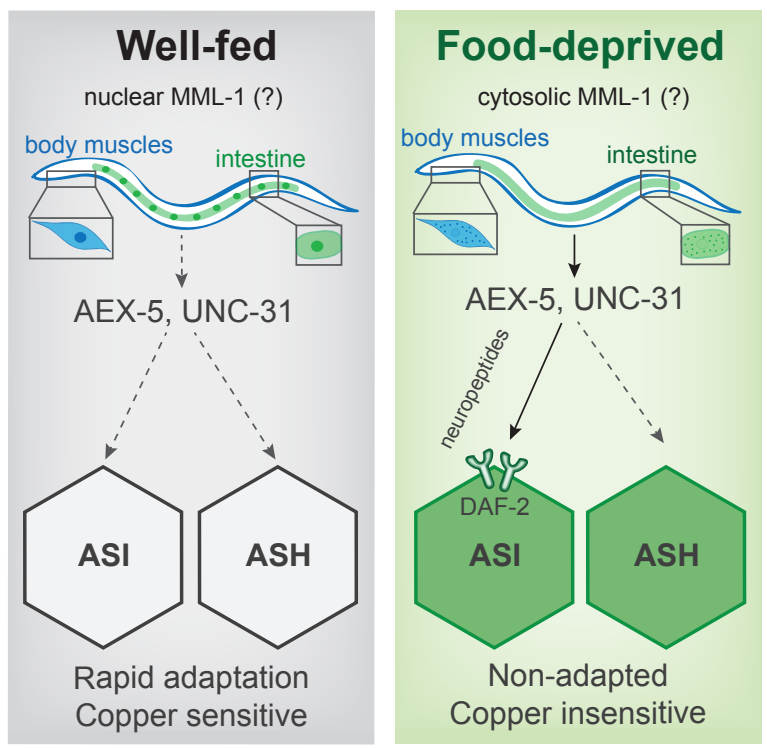

